\title{
Achievable rates optimization for broadcast channels using finite size constellations under transmission constraints
}

\author{
Zeina Mheich ${ }^{1,2,3}$, Florence Alberge ${ }^{1,2,3^{*}}$ and Pierre Duhamel ${ }^{1,2,3}$
}

\begin{abstract}
In this paper, maximal achievable rate regions are derived for power-constrained AWGN broadcast channel involving finite constellations and two users. The achievable rate region is studied for various transmission strategies including superposition coding and compared to standard schemes such as time sharing. The maximal achievable rates are obtained by optimizing over both the joint distribution of probability and over the constellation symbol positions. A numerical solution is proposed for solving this non-convex optimization problem. Then, we consider several variations of the same problem by introducing various constraints on the optimization variables. The aim is to evaluate efficiency vs. complexity tradeoffs of several transmission strategies, some of which (the simplest ones) can be found in actual standards. The improvement for each scheme is evaluated in terms of SNR savings for target achievable rates or/and percentage of gain in achievable rates for one user compared to a reference scheme. As an application, two scenarios of coverage areas and user alphabets are considered. This study allows to evaluate with practical criteria the performance improvement brought by more advanced schemes.
\end{abstract}

Keywords: AWGN broadcast channels; Achievable rate region; Hierarchical modulation; Superposition modulation; Superposition coding; Constellation shaping; Non-convex optimization

\section{Introduction}

During the past few decades, information networks have witnessed tremendous and rapid advances, based on the important growth in the adoption of new wireless technologies, applications and services, first from cellular networks and more recently for computer networks (WLANs). Consequently, wireless networks are exposed to capacity and coverage problems, and the focus is now shifting towards capturing some of the aspects of realistic networks by studying natural network models such as models with broadcasting.

In 1972, achievable rate region is obtained by Cover in [1] for Gaussian broadcast channels with two outputs and generalized by Bergmans to broadcast channels with any number of outputs [2]. Roughly a year later, the optimality of the sets of achievable rates was established

\footnotetext{
*Correspondence: alberge@lss.supelec.fr

1 University Paris-Sud, UMR8506 Orsay, F-91405, France

${ }^{2}$ CNRS, Gif-sur-Yvette, F-91192, France

Full list of author information is available at the end of the article
}

by Bergmans [3] and Gallager [4]. Superposition coding is a possible solution to achieve good rate regions in which information intended for high-noise receivers and information intended for low-noise receivers are superimposed and transmitted simultaneously on the same radio resource. The low-noise receivers can always decode messages intended for the high-noise receivers. Thus, they effectively cancel out the interference due to the signal intended for the high-noise receivers, and then decode their own message. The high-noise receivers decode their messages by treating the low-noise receivers message as noise. Superposition coding appears in several contexts in information theory and is closely related to multilevel coding and unequal error protection [5,6]. Cover showed [1] that the superposition coding reaches the theoretical limit of the capacity region for two user Gaussian broadcast channel using an infinite Gaussian input alphabet for each user. A treatment of the case of

\section{Springer}

(c) 2013 Mheich et al: licensee Springer. This is an Open Access article distributed under the terms of the Creative Commons Attribution License (http://creativecommons.org/licenses/by/2.0), which permits unrestricted use, distribution, and reproduction in any medium, provided the original work is properly cited. 
multiple transmitter/receivers for the band-limited additive white Gaussian noise channel is given by Bergmans and Cover in [7], where it is proved that superposition coding can achieve higher-rate region than orthogonal schemes such as frequency-division multiple access (FDMA) or time division multiple access (TDMA). However, in actual transmission systems, the channel input is constrained to a finite size alphabet with equal probability symbols. A well-known practical implementation of superposition coding is hierarchical modulation, also called layered modulation, which uses constellations with non-uniformly spaced signal points creating different levels of error protection. Hierarchical modulation is used to mitigate the cliff effect in digital television broadcast and is included in various standards, such as Digital Video Broadcast for Terrestrial Television (DVB-T) [8], DVB to Handhelds (DVB-H), and DVB Satellite services to Handhelds (DVB-SH) [9] standard proposal for mobile digital TV transmission. A study about the performance of hierarchical modulation and a comparison with time sharing strategy in terms of achievable rates can be found in [10].

The restriction imposed by practical systems in using finite signaling constellation and equiprobable symbols reduces the achievable rates and leads to a gap with the capacity region achieved with Gaussian input alphabets for AWGN broadcast channel. This gap can be reduced using a technique called constellation shaping. In fact, most results for constellation shaping with finite signal constellations consider only point-to-point communication systems [11]. Then, the concept of constellation shaping has been adapted to most modern coding and modulation techniques as for example turbo coding and BICM schemes [12-19]. For broadcast channels, the achievable rate region for two-user AWGN broadcast channels with finite input alphabets is derived in [20] when superposition of modulated signal is used as transmission strategy. In their work, the authors assume a uniform distribution over the finite input set. To our knowledge, no study is available about the maximization of the achievable rate region for two-user AWGN broadcast channels with finite size constellations by optimizing over both the joint probability distribution and constellation symbol positions for a broadcast transmission strategy. This general framework encompasses hierarchical modulations as a special case. In this paper, maximal achievable rate regions are derived for powerconstrained AWGN broadcast channel of two users with $M$-pulse amplitude modulation ( $M$-PAM) constellations of $M$ points using various transmission strategies. A numerical solution is proposed for solving this nonconcave optimization problem. In a typical broadcast system, there is a trade off between achievable rates and coverage areas. Therefore, we are interested in determining the transmission strategy which provides the best achievable rates or the maximal SNR gain for a given coverage scenario. The compromise between the simplicity of implementation and expected gains is also evaluated.

The organization of the paper is as follows. Section 2 recalls some information theory results on broadcast channels and degraded broadcast channels. In section 3, various transmission strategies for broadcast systems are described. Section 4 gives a formulation of the problem in terms of optimization for the various transmission strategies under consideration. Then, computational aspects are discussed. An iterative algorithm is proposed for the computation of maximal achievable rate regions using superposition coding (general case) and M-PAM constellation or in the particular case of superposition modulation. The proposed algorithm can handle an optimization with respect to the joint distribution of probability or with respect to the positions of constellation symbols. Both variables can also be considered jointly. Obviously, the best results are obtained for the most general case. Our target is to (1) evaluate the loss experienced using simple schemes, (2) identify situations in which complex schemes (non-standard) lead to significant improvements. As an application, we consider, in section 5, several scenarios of coverage areas and user alphabets, and we give conclusions about the transmission strategies which can provide the best trade off between efficiency and complexity of implementation.

\section{AWGN broadcast channels}

A two-receiver (users) broadcast channel (BC) consists of an input alphabet $\mathcal{X}$, two output alphabets $\mathcal{Y}_{1}$ (user 1 ), $\mathcal{Y}_{2}$ (user 2), and a conditional pdf $P_{Y_{1} Y_{2} \mid X}$ on $\mathcal{Y}_{1} \times \mathcal{Y}_{2}$. Let $X, Y_{1}$, and $Y_{2}$ be random variables representing the input and outputs of the BC. Figure 1 depicts the two users $\mathrm{BC}$ with two independent messages $W_{1}$ and $W_{2}$. The encoder generates a codeword $x^{n}\left(w_{1}, w_{2}\right)$ of length $n$ based on these two messages. Each user receives, respectively, $y_{1}^{n}$ and $y_{2}^{n}$. A BC is said to be physically degraded if $P_{Y_{1} Y_{2} \mid X}\left(y_{1}, y_{2} \mid x\right)=P_{Y_{1} \mid X}\left(y_{1} \mid x\right) \cdot P_{Y_{2} \mid Y_{1}}\left(y_{2} \mid y_{1}\right)$ (i.e., $X \rightarrow$ $Y_{1} \rightarrow Y_{2}$ form a Markov chain). A BC is said to be stochastically degraded or degraded if there exists a random variable $\tilde{Y}_{1}$ which has the same conditional pdf as $Y_{1}$ given $X$, such that $X \rightarrow \tilde{Y}_{1} \rightarrow Y_{2}$ forms a Markov chain. We are interested in degraded BC because its capacity

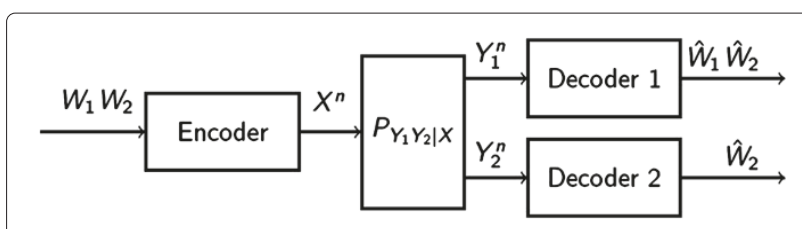

Figure 1 The two-user broadcast channel. 
region is known, while it is not available for the general case.

In our system model, $W_{1}$ denotes the private message intended for receiver 1 only, and $W_{2}$ is a common message for both receivers. A typical example of this situation is digital TV broadcasting to two different groups of receivers, classified according to their channel conditions, where the basic signal (common signal) should be available to all receivers. The higher quality is realized by adding the basic signal with an incremental signal (private signal for receivers of good channel conditions) which carries TV signal with a high data rate, such as HDTV.

Let $R_{1}$ and $R_{2}$ be the rates at which the transmitter is sending $W_{1}$ and $W_{2}$, respectively. Thus, user 1 achieves $R_{1}+R_{2}$, while user 2 achieves $R_{2}$. The capacity region of the degraded broadcast channel $X \rightarrow Y_{1} \rightarrow Y_{2}$ in Figure 1 is the convex hull of the closure of rate pairs $\left(R_{1}+R_{2}, R_{2}\right)$ satisfying

$$
\begin{aligned}
& R_{1} \leq I\left(X ; Y_{1} \mid U\right) \\
& R_{2} \leq I\left(U ; Y_{2}\right)
\end{aligned}
$$

for some joint distribution $P_{U X Y_{1} Y_{2}}=P_{U X} \cdot P_{Y_{1} \mid X} \cdot P_{Y_{2} \mid X}$ on $\left\{\mathcal{U} \times \mathcal{X} \times \mathcal{Y}_{1} \times \mathcal{Y}_{2}\right\}$ [21]. $P_{Y_{1} \mid X}$ and $P_{Y_{2} \mid X}$ are conditional pdfs that depend on the channel model. $P_{U X}$ is the joint probability distribution of $U$ and $X$, where the auxiliary random variable $U$ has cardinality bounded by $|\mathcal{U}| \leq \min \left\{|\mathcal{X}|,\left|\mathcal{Y}_{1}\right|,\left|\mathcal{Y}_{2}\right|\right\}$. The capacity region is achieved using superposition coding, where $U$ serves as the center of a cloud of codewords that can be distinguished by both receivers. Since the capacity region of a BC depends only on the conditional marginals, the capacity region of the stochastically degraded $B C$ is equal to that of the corresponding physically degraded channel. Cover [1] showed that in the case of binary symmetric BC and AWGN BC, superposition coding expands the rate region beyond that achievable with time sharing.

Now, consider the Gaussian broadcast channel with two users. Without loss of generality, assume that $Y_{1}$ is less noisy than $Y_{2}$. It can easily be shown that scalar Gaussian broadcast channels are equivalent to a degraded channel,

$$
\begin{aligned}
& Y_{1}=X+Z_{1} \\
& Y_{2}=X+Z_{2}=Y_{1}+Z_{2}^{\prime},
\end{aligned}
$$

where $Z_{1} \sim \mathcal{N}\left(0, \sigma_{1}^{2}\right), Z_{2} \sim \mathcal{N}\left(0, \sigma_{2}^{2}\right), Z_{2}^{\prime} \sim \mathcal{N}\left(0, \sigma_{2}^{2}-\right.$ $\left.\sigma_{1}^{2}\right)$, and $Z_{1}, Z_{2}^{\prime}$ are independent. Thus, Gaussian BC is stochastically degraded. We assume an average power constraint on the transmitted power $P$ defined as $\mathbb{E}\left[X^{2}\right] \leq$ $P$. The received signal to noise ratio for each user is $\mathrm{SNR}_{i}=\frac{P}{\sigma_{i}^{2}}$, where $\mathrm{SNR}_{1}>\mathrm{SNR}_{2}$, and $\sigma_{i}^{2}$ is the variance of the noise $Z_{i}$. The capacity region of the AWGN-BC is the set of rate pairs $\left(R_{1}+R_{2}, R_{2}\right)$, such that

$$
\begin{aligned}
& R_{1} \leq C\left(\alpha \cdot \mathrm{SNR}_{1}\right) \\
& R_{2} \leq C\left(\frac{(1-\alpha) \cdot \mathrm{SNR}_{2}}{\alpha \cdot \mathrm{SNR}_{2}+1}\right)
\end{aligned}
$$

for all $\alpha \in[0,1]$, where $C(x)=\frac{1}{2} \cdot \log _{2}(1+x)$. The theoretical limit of two-user AWGN BC is achieved using signal superposition [1].

\section{Broadcast transmission strategies}

In this section, various transmission strategies for broadcast systems are described. The strategies are presented in ascending order of implementation complexity. Specifically, by moving from one strategy to another, we release some constraints on the system implementation to reach finally the most complex strategy that can be used to broadcast information for users. Obviously, since the simple schemes can be understood as adding constraints to the most general case, they are less efficient in terms of attainable rates.

\subsection{Time sharing}

Time sharing (TS) has been widely used in broadcast systems as broadcast transmission strategy. In time sharing scheme, a percentage of time is used to send one message, and the rest of the time is used to send another message. Thus, it is practical to implement because the rate pairs can be achieved by strategies used for point-to-point channel and sharing the time between messages. As in previous works on broadcasting, this situation serves as a reference for the more advanced schemes.

In this work, a time sharing scheme with standard constellation $M$-PAM (Figure 2) is considered when symbols are used with equal probability. A standard $M$-PAM constellation is defined as a constellation with $M$ real symbols belonging to $\mathcal{X}=\{M-1-2 \cdot(i-1)$, for $i=1, \ldots, M\}$. During the time slot dedicated to send a message, only one data stream is sent using the entire set of constellation points. In classical implementations of time sharing,

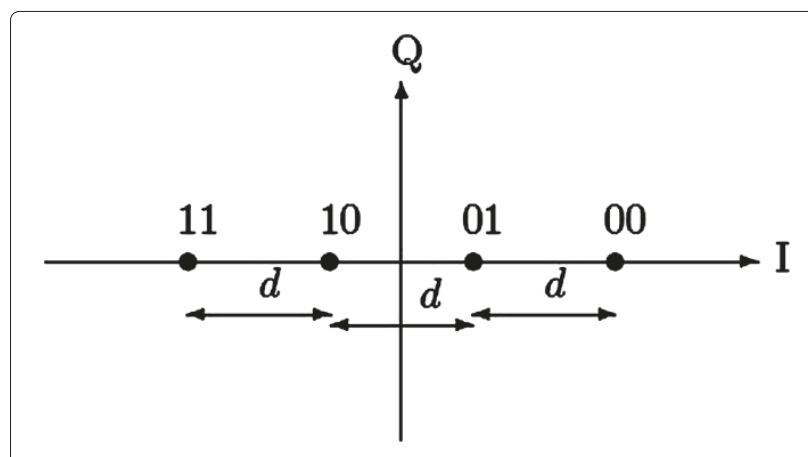

Figure 2 4-PAM with equally spaced symbols. 
the conventional $M$-PAM symbols are equally spaced and used with equal probability.

\subsection{Hierarchical Modulation (HM)}

In two-layer hierarchical modulation, constellation symbols are used to transmit two data streams simultaneously for two users [22,23]. Constellation symbols are usually chosen with the same probability but may be non-equally spaced. These symbols can be considered as the sum of two lower-order modulations, one for each user. The modulation with higher power is used for the 'bad' channel, the one with smallest power for the 'good' channel. Hence, the encoding using hierarchical modulation can be separable for the two streams which is more practical.

This is explained here using 4-PAM as an example. Figure 3 shows the constellation diagram of a hierarchical 4-PAM with parameter $\ell=\ell_{1} / \ell_{2}$ used to determine the spacing between the groups of constellation points (clouds). $\ell$ is the ratio of the spacing between the groups to the spacing between individual points within a group. Standard values of $\ell$ are 1,2 , and 4. When $\ell$ increases, with a fixed total transmission power $P$, the two points from both sides of origin form a cloud. The location of a point within its cloud is regarded as the information for the 'good' user. The other information, i.e., the number of the cloud in which the point is located is the information for the 'bad' user. In this way, two separate data streams can be made available for transmission. Formally, we are still dealing with 4-PAM, but in the hierarchical interpretation, it is viewed as the combination of two BPSK modulations which have different robustness to noise. In other words, the service coverage areas differ in size for both users. The better-protected data stream is referred to as the high-priority (HP) stream which is mapped in Figure 3 to the most significant bit. The other one is referred to as the low-priority (LP) stream (Figure 3 ) and mapped in Figure 3 to the least significant bit. Receivers

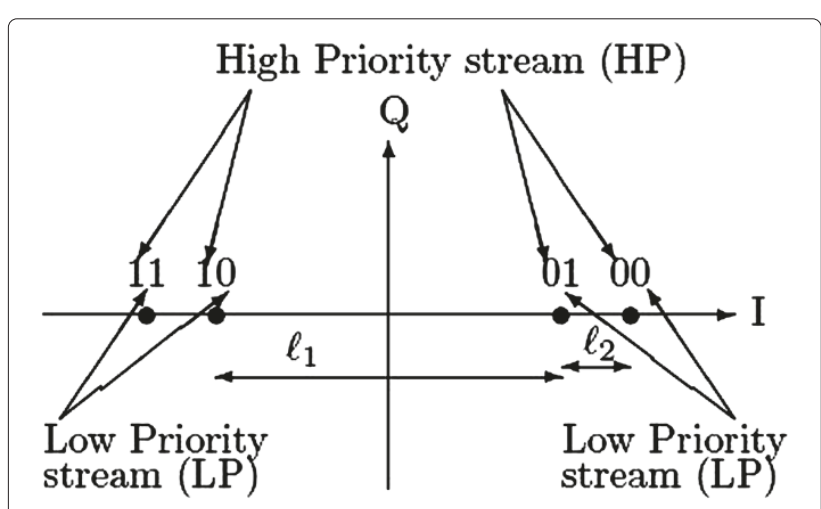

Figure 3 Hierarchical 4-PAM with parameter $\ell=\ell_{1} / \ell_{2}$. with good reception conditions can receive both streams, while those with poorer reception conditions may only receive the high priority stream considering the LP stream as noise. This corresponds to a specific labeling of the modulation.

\subsection{Superposition modulation}

In superposition modulation (SM) [24], the $M$ constellation points are used such that the labeling is separable, i.e., $M=M_{1} M_{2}$, and that the $M$ points are obtained by adding (in $\mathbb{R}$ ) two rv's $X_{1}$ and $X_{2}$ of cardinality $M_{1}$ and $M_{2}$, respectively $\left(M_{1}, M_{2} \in \mathbb{N} \backslash\{0,1\}\right)$. Thus, this scheme is with an enlarged set of feasible labelings than in the previous case $[25,26]$. This leads also to $U \equiv X_{2}$ for superposition modulation because user 2 can distinguish only $U$.

This work studies several cases of superposition modulation. First, when the constellation symbols for each user are used with equal probability. This case will be

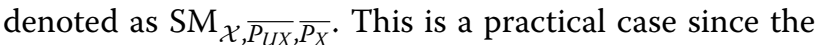
encoding of the messages is separable, and the symbols are used with equal probability as in real transmission systems. Then, the constraint of using equiprobable symbols is released and the symbols of user constellations can be dependent and used with non-equal probability $\left(P_{U X}\right.$ non-uniform). Thus, the encoding here is done jointly for the two messages. This strategy will be denoted $\mathrm{SM}_{\overline{\mathcal{X}}, P_{U X}, P_{X}}$ when the symbols take the values of a stan-

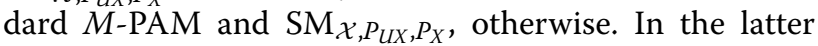
case, the symbol positions can take arbitrary values and will be considered as variables to be optimized. The definition of superposition modulation can be generalized using more general form for $P_{U X}$ than the uniform case. In superposition modulation, $2^{n R_{2}}$ independent codewords $u^{n}=x^{(2) n}\left(w_{2}\right)$ of length $n$ are generated according to $P_{U}$; for each of these codewords, $2^{n R_{1}}$ satellite codewords $v^{n}=x^{(1) n}\left(w_{1}\right)$ are generated and added to form codewords $x^{n}\left(w_{1}, w_{2}\right)=u^{n}+v^{n}$ according to $P_{X \mid U}$. Thus, the fine information $v^{n}$ is superimposed on the coarse information $u^{n}$.

Note that the capacity region of Gaussian broadcast channel is achieved using this coding scheme and successive cancellation decoding, where $U\left(\equiv X_{2}\right)$ and $V\left(\equiv X_{1}\right)$ are independent random variables following normal distributions. However, we do not assume here that $U$ and $V$ are independent. Consequently, for superposition modulation, $P_{U X}$ takes a specific expression. As an example, consider an 8-PAM modulation. In that case, the transmitted signal at time $k$ is the sum of the two users signals and is given by $x_{k}=x_{k}^{(1)}+x_{k}^{(2)}$, where $x_{k}^{(1)} \in \mathcal{X}_{1}$ and $x_{k}^{(2)} \in \mathcal{X}_{2}$ with $M_{1} \cdot M_{2}=8$. Two configurations are possible either $M_{2}=4\left(\mathcal{X}_{1}\right.$ is a BPSK, and $\mathcal{X}_{2}$ is a 4 PAM) or $M_{2}=2\left(\mathcal{X}_{1}\right.$ is a 4-PAM, and $\mathcal{X}_{2}$ is a BPSK). In 
both cases, $P_{U X}$ is a sparse matrix of size $M_{2} \times M$ with expression

$$
P_{U X}=\left[\begin{array}{cccccccc}
p_{00} & p_{01} & 0 & 0 & 0 & 0 & 0 & 0 \\
0 & 0 & p_{12} & p_{13} & 0 & 0 & 0 & 0 \\
0 & 0 & 0 & 0 & p_{24} & p_{25} & 0 & 0 \\
0 & 0 & 0 & 0 & 0 & 0 & p_{36} & p_{37}
\end{array}\right] \text { if } M_{1}=2, M_{2}=4
$$

$$
P_{U X}=\left[\begin{array}{cccccccc}
p_{00} & p_{01} & p_{02} & p_{03} & 0 & 0 & 0 & 0 \\
0 & 0 & 0 & 0 & p_{14} & p_{15} & p_{16} & p_{17}
\end{array}\right] \text { if } M_{1}=4, M_{2}=2
$$

where $P_{U X}[i, j]=p_{i-1, j-1}=\operatorname{Pr}\left\{U=u_{i-1}, X=x_{j-1}\right\}$. In both cases, the number of elements to be computed is 8 .

Note also that $P_{U X}$ and $\mathcal{X}$ (of cardinality $M$ ) determine the labeling of the input signal constellation for a fixed labeling for $\mathcal{X}_{1}$ and $\mathcal{X}_{2}$ [25,26]. Thus, the information can be distinguished using the labeling. Consider for example a label $l_{k}^{u}$ of $\log _{2}\left(\left|\mathcal{X}_{2}\right|\right)$ binary labels for $u_{k}$ and $l_{j}^{v}$ of $\log _{2}\left(\left|\mathcal{X}_{1}\right|\right)$ binary labels for $v_{j}$ with $k \in\left\{0, . .,\left|\mathcal{X}_{2}\right|-1\right\}$ and $j \in\left\{0, . .,\left|\mathcal{X}_{1}\right|-1\right\}$. Obviously, the $M$ symbols $x_{i}, i \in$ $\{0, . .,|\mathcal{X}|-1\}$ carry $\log _{2}(M)$ binary labels which are the concatenations of the labels of $u_{k}$ and $v_{j}$ such as $x_{i}=$ $u_{k}+v_{j}$.

Part of this work on superposition modulation was presented in [25-27], where the achievable rate regions for $\mathrm{SM}_{\mathcal{X}, \overline{P_{U X}}, \overline{P_{X}}}$ and $\mathrm{SM}_{\mathcal{X}, P_{U X}, P_{X}}$ strategies are analyzed using a 4-PAM constellation in $[25,26]$ and for $\{4,8,16\}$ PAM constellations in [27]. In this work, the achievable rates are also derived for $\mathrm{SM}_{\overline{\mathcal{X}}, P_{U X}, P_{X}}$ using $\{4,8,16\}$-PAM constellations.

\subsection{Superposition coding}

Superposition coding (SC) is one of the basics of coding schemes in network information theory. This idea was first introduced by Cover in an information theoretic study of broadcast channels [1]. In superposition coding, the joint distribution of probability $P_{U X}$ can take a more general form than in the case of superposition modulation. In this case, the labeling cannot distinguish between the common information and the private information for user 1, a fact which increases the decoder complexity. Indeed, since the auxiliary random variable $U$ has cardinality bounded by $|\mathcal{U}| \leq \min \left\{|\mathcal{X}|,\left|\mathcal{Y}_{1}\right|,\left|\mathcal{Y}_{2}\right|\right\}$, we use the name general superposition coding or superposition coding simply to describe the case, where $|\mathcal{U}|=$ $\min \left\{|\mathcal{X}|,\left|\mathcal{Y}_{1}\right|,\left|\mathcal{Y}_{2}\right|\right\}$. For superposition coding and with $M$-PAM modulation, $P_{U X}$ is an $M \times M$ matrix with elements $p_{i, j}$.

The basics of superposition coding are briefly recalled below; a detailed description is given in [28]. In this scheme, $2^{n R_{2}}$ sequences $u^{n}\left(w_{2}\right), w_{2} \in\left[1,2^{n R_{2}}\right]$ each i.i.d., are generated randomly and independently to represent the coarse message, each according to $\prod_{i=1}^{n} p_{U}\left(u_{i}\right)$. For each auxiliary sequence, $u^{n}\left(w_{2}\right)$ randomly, conditionally, and independently generates $2^{n R_{1}}$ sequences $x^{n}\left(w_{1}, w_{2}\right)$ and $w_{1} \in\left[1,2^{n R_{1}}\right]$, each according to $\prod_{i=1}^{n} p_{X \mid U}\left(x_{i} \mid u_{i}\left(w_{2}\right)\right)$ to represent the fine message $w_{1}$. Thus, in superposition coding, the auxiliary random variable $U$ serves as a cloud center for the information, distinguishable by both receivers. In this case, the decoding of information by users is based on large block joint typicality. This comes in contrast with the simpler cases where the message for user 2 was carried by the center of modulation clouds which imply a possible scalar detection.

The achievable rates for superposition coding will be studied for various strategies corresponding to different constraints on $P_{U X}$ and/or $\mathcal{X}$. An exhaustive list of all the strategies under consideration is given in Table 1, where redundant configurations are omitted.

\section{Achievable rate regions}

For a two-user Gaussian BC, the theoretical limit of the capacity region is achieved using Gaussian input alphabet for each user. However, practical implementation constraints impose the use of finite input alphabets, and the symbols are usually chosen with equal probability. These restrictions contribute to increase the gap between the capacity region achieved with infinite Gaussian inputs and the throughput obtained in practical situations. In this section, we are interested in computing the achievable rate region of power-constrained AWGN BC when the transmitted signal is modulated using an $M$-PAM constellation, under the various situations described above. Since the last case (superposition coding) encompasses all previous ones as special cases, the corresponding optimization problems can be solved with the same strategy, which is detailed in this section.

\begin{tabular}{|c|c|c|c|}
\hline Transmission & Variables & Constraints & Designation \\
\hline SM & $\mathcal{X}$ & Uniform distribution for $P_{U x}$ & $\bar{S} \mathrm{SM}_{\mathcal{X}, \overline{P_{u X}, \overline{P_{X}}}}$ \\
\hline SM & $P_{U X X}$ s.t. $\sum_{i, j} p_{i, j}=1$ & Symbol locations: M-PAM & $S M_{\overline{\mathcal{X}}, P_{U X}, P_{X}}$ \\
\hline \multirow[t]{2}{*}{ SM } & $\mathcal{X}$ & & $S M_{\mathcal{X}, P_{U X}, P_{X}}$ \\
\hline & $P_{U X X}$ s.t. $\sum_{i, j} p_{i, j}=1$ & & \\
\hline \multirow[t]{2}{*}{ SC } & $P_{U X}$ s.t. $\sum_{i} p_{i, j}=\frac{1}{M}$ & Symbol locations: M-PAM & $\mathrm{SC}_{\overline{\mathcal{X}}, P_{U X}, \overline{P_{X}}}$ \\
\hline & & Uniform distribution for $P_{X}$ & \\
\hline \multirow[t]{2}{*}{ SC } & $\mathcal{X}$ & Uniform distribution for $P_{X}$ & $S C_{\mathcal{X}, P_{U X}, \overline{P_{X}}}$ \\
\hline & $P_{U X}$ s.t. $\sum_{i} p_{i, j}=\frac{1}{M}$ & & \\
\hline SC & PUX s.t. $\sum_{i, j} p_{i, j}=1$ & Symbol locations: M-PAM & $S C_{\overline{\mathcal{X}}, P_{U X}, P_{X}}$ \\
\hline \multirow[t]{2}{*}{ SC } & $\mathcal{X}$ & & $\mathrm{SC}_{\mathcal{X}, P_{U X}, P_{X}}$ \\
\hline & $P_{U X}$ s.t. $\sum_{i, j} p_{i, j}=1$ & & \\
\hline
\end{tabular}

\section{Table 1 Strategies under consideration}




\subsection{Problem formulation}

Consider a two-user memoryless AWGN broadcast channel $\left(\mathrm{SNR}_{1}>\mathrm{SNR}_{2}\right)$ with signal power constraint $P$. The channel input belongs to a finite set $\mathcal{X}=\left\{x_{0}, \ldots, x_{M-1}\right\} \subset$ $\mathbb{R}$ represented by an $M$-PAM constellation. Assume a symmetric input signal constellation with respect to the origin. Since $\mathcal{U}$ has cardinality bounded by $|\mathcal{U}| \leq$ $\min \left\{|\mathcal{X}|,\left|\mathcal{Y}_{1}\right|,\left|\mathcal{Y}_{2}\right|\right\}$, and the output alphabet cardinality for an AWGN channel is infinite, we have $|\mathcal{U}| \leq|\mathcal{X}|$. Thus, $|\mathcal{U}| \leq M$.

To determine the maximal achievable rate region using superposition coding, consider the case $|\mathcal{U}|=M$. For superposition modulation, we take into account the specificity on $P_{U X}$ given in section 3.3. We also consider within the same framework the problem of maximizing the achievable rates under additional constraints on optimization variables $\left(P_{U X}\right.$ and $\left.\mathcal{X}\right)$ : standard $M$-PAM symbols values, uniform distribution for $P_{U X}$, uniform distribution for $P_{X}$. The problem of maximizing the achievable rates under a specific situation is solved subject to a combination of constraints according to Table 1 . We recall that in this work, message $w_{2}$ is a common message to both receivers, and $w_{1}$ is a private message to user 1 . Thus, the achievable rate region $\left(R_{2}\right.$ vs. $\left.R_{1}+R_{2}\right)$ can be obtained by solving the weighted sum rate $\left(\theta \cdot R_{1}+(1-\theta) \cdot R_{2}\right)$ maximization for $\theta \in[0,0.5]$. Indeed, for $\theta=0$, we maximize the common information rate $R_{2}$, and when $\theta=0.5$, we maximize the rate achieved by user $1\left(R_{1}+R_{2}\right)$. Using (1) and (2), the optimization problem under consideration is:

$$
\begin{array}{ll}
\max _{P_{U X}, \mathcal{X}} & \theta \cdot I\left(X ; Y_{1} \mid U\right)+(1-\theta) \cdot I\left(U ; Y_{2}\right) \\
\text { s.t. } & p_{i j} \geq 0 \quad \forall i, j \\
& \sum_{i, j} p_{i j} \cdot x_{j}^{2} \leq P
\end{array}
$$

and subject to the constraint on the joint pdf $P_{U X}$ or on $\mathcal{X}$ given in Table 1 for each strategy, where $p_{i j}=\operatorname{Pr}\{U=$ $\left.u_{i}, X=x_{i}\right\}, j \in\{0, . ., M-1\}$, and $i \in\{0, . .,|\mathcal{U}|-1\}$. The two mutual information $I\left(X ; Y_{1} \mid U\right)$ and $I\left(U ; Y_{2}\right)$ can be written as follows:

$$
\begin{aligned}
I\left(X ; Y_{1} \mid U\right)= & \sum_{i, j} \int_{-\infty}^{+\infty} p_{i j} P_{Y_{1} \mid X}\left(y_{1} \mid x_{j}\right) \\
& \times \log \frac{\left(\sum_{j^{\prime}} p_{i j^{\prime}}\right) P_{Y_{1} \mid X}\left(y_{1} \mid x_{j}\right)}{\sum_{j^{\prime}} p_{i j^{\prime}} P_{Y_{1} \mid X}\left(y_{1} \mid x_{j^{\prime}}\right)} d y_{1} \\
I\left(U ; Y_{2}\right)= & \sum_{i} \int_{-\infty}^{+\infty}\left(\sum_{j} p_{i j} P_{Y_{2} \mid X}\left(y_{2} \mid x_{j}\right)\right) \\
& \times \log \frac{\sum_{j^{\prime}} p_{i j^{\prime}} P_{Y_{2} \mid X}\left(y_{2} \mid x_{j^{\prime}}\right)}{\left(\sum_{j^{\prime}} p_{i j^{\prime}}\right)\left(\sum_{i^{\prime}, j^{\prime}} p_{i^{\prime} j^{\prime}} P_{Y_{2} \mid X}\left(y_{2} \mid x_{j^{\prime}}\right)\right)} d y_{2},
\end{aligned}
$$

where all logarithms are taken base 2 . The AWGN channel for each user is characterized by the conditional pdf

$$
P_{Y_{i} \mid X}(y \mid x)=\frac{1}{\sqrt{2 \pi \sigma_{i}^{2}}} \cdot e^{-\frac{(y-x)^{2}}{2 \sigma_{i}^{2}}} i \in\{1,2\} .
$$

When $\theta=0$ or $\theta=1$ and for $|\mathcal{U}|=M$ (which are referred in this paper as point-to-point (PtP) channel case), the individual achievable rates $R_{2}$ and $R_{1}$ are maximized respectively. The problem (9) is equivalent to

$$
\begin{array}{ll}
\max _{P_{X}, \mathcal{X}} & I\left(X ; Y_{k}\right) \\
\text { s.t. } & p_{i} \geq 0 \quad \forall i \\
& \sum_{i} p_{i}=1 \\
& \sum_{i} p_{i} \cdot x_{i}^{2} \leq P,
\end{array}
$$

where $p_{i}=\operatorname{Pr}\left\{X=x_{i}\right\}, i \in\{0, . ., M-1\}$ is the input probability distribution, and $k \in\{1,2\}$. When $\theta=0$ or 1 , problem (13) is solved for $k=2$ and 1 , respectively, with $I\left(X ; Y_{k}\right)$ given by

$$
\begin{aligned}
I\left(X ; Y_{k}\right)= & \int_{-\infty}^{+\infty} \sum_{j} p_{j} P_{Y_{k} \mid X}\left(y_{k} \mid x_{j}\right) \\
& \times \log \frac{P_{Y_{k} \mid X}\left(y_{k} \mid x_{j}\right)}{\sum_{j^{\prime}} p_{j^{\prime}} P_{Y_{k} \mid X}\left(y_{k} \mid x_{j^{\prime}}\right)} d y_{k} .
\end{aligned}
$$

For the time sharing scheme using standard constellation, the achievable rate pair $\left(R_{1}+R_{2}, R_{2}\right)$ is such that [1]

$$
\left\{\begin{array}{l}
R_{1}=\alpha \overline{R_{1}} \\
R_{2}=(1-\alpha) \overline{R_{2}}
\end{array},\right.
$$

where $\overline{R_{1}}$ and $\overline{R_{2}}$ are achievable rates for PtP channel using standard $M$-PAM constellation at $\mathrm{SNR}_{1}$ and $\mathrm{SNR}_{2}$, respectively. Varying $\alpha$ from 0 to 1 yields achievable rate region.

Problem (9) is not convex; therefore, direct numerical optimization is inefficient. Clearly, an exhaustive search is not feasible as the complexity would be exponential in the total number of variables. An iterative method for solving (9) is proposed in the next section.

\subsection{Numerical solution}

Consider a regularized version of (9) as

$$
\begin{aligned}
L\left(P_{U X}, x_{0}, . ., x_{M-1}, s\right)= & \theta \cdot I\left(X ; Y_{1} \mid U\right)+(1-\theta) \cdot I\left(U ; Y_{2}\right) \\
& +s \cdot\left(P-\sum_{i=0}^{|\mathcal{U}|-1} \sum_{j=0}^{M-1} p_{i j} \cdot x_{j}^{2}\right),
\end{aligned}
$$

where $s$ is a regularization parameter. For a given value of $s$, the optimization problem in (16) is solved (for the most general case) with respect to $P_{U X}$ and to $\mathcal{X}=$ $\left(x_{0}, x_{1}, \ldots, x_{M-1}\right)$ alternately until convergence:

$$
P_{U X}^{(\ell)}=\arg \max _{P_{U X} \in \mathcal{C}} L\left(P_{U X}, x_{0}^{(\ell-1)}, . ., x_{M-1}^{(\ell-1)}, s\right)
$$




$$
\mathcal{X}^{(\ell)}=\arg \max _{\mathcal{X}} L\left(P_{U X}^{(\ell)}, x_{0}, . ., x_{M-1}, s\right),
$$

where $\ell$ is the iteration index, and $\mathcal{C}$ denotes the set of constraints on $P_{U X}$ and can be defined either as $\mathcal{C}=\left\{P_{U X}\right.$ : $\left.p_{i j} \geq 0, \sum_{i, j} p_{i, j}=1\right\}$ or as $\mathcal{C}=\left\{P_{U X}: p_{i j} \geq 0, \sum_{i} p_{i, j}=\frac{1}{M}\right\}$ (equiprobable symbols). The optimization problem in (17) with constraint set $\mathcal{C}=\left\{P_{U X}: p_{i j} \geq 0, \sum_{i, j} p_{i, j}=1\right\}$ can be handled by a modified 'Blahut-Arimoto'-type algorithm [29]. Indeed, in order to take into account the regularization, we can show that the Blahut-Arimototype algorithm proposed in [30] for broadcast channels should be modified by replacing Equation (19) of Lemma 3 in [30] by $q^{*}(u, x)=\frac{\beta[Q, \tilde{Q}, \bar{Q}](u, x) \cdot e^{-s \frac{x^{2}}{1-\theta}}}{\sum_{u^{\prime}, x^{\prime}} \beta[Q, \tilde{Q}, \bar{Q}]\left(u^{\prime}, x^{\prime}\right) \cdot e^{-s \frac{x^{\prime 2}}{1-\theta}}}$ instead of $q^{*}(u, x)=\frac{\beta[Q, \tilde{Q}, \bar{Q}](u, x)}{\sum_{u^{\prime}, x^{\prime}} \beta[Q, \tilde{Q}, \bar{Q}]\left(u^{\prime}, x^{\prime}\right)}$, where $\beta[Q, \tilde{Q}, \bar{Q}](u, x)$ is defined in Equation (19) of [30]. When there is an additional constraint on constellation symbols to be equiprobable, i.e., $\mathcal{C}=\left\{P_{U X}: p_{i j} \geq 0, \sum_{i, j} p_{i, j}=1\right.$ and $\sum_{i} p_{i, j}=$ $\left.\frac{1}{M}\right\}$, the Blahut-Arimoto-type algorithm in [30] should also be modified to take into account the additional constraint. In this case, Equation (19) of Lemma 3 in reference [30] should be replaced by $q^{*}(u, x)=\frac{1}{|\mathcal{X}|} \cdot \frac{\beta[Q, \tilde{Q}, \bar{Q}](u, x)}{\sum_{u} \beta[Q, \tilde{Q}, \bar{Q}](u, x)}$, which does not depend on $s$, where $\beta[Q, \tilde{Q}, \bar{Q}](u, x)$ is defined in Equation (19) in this reference.

Now consider (18). The function $L\left(P_{U X}^{(\ell)}, x_{0}, . ., x_{M-1}, s\right)$ is not a concave function for all $\mathcal{X} \in \mathbb{R}^{M}$. However, we observed in our experiments that $L\left(P_{U X}^{(\ell)}, x_{0}, . ., x_{M-1}, s\right)$ is a concave function if $\mathcal{X} \in \mathcal{D}$, where $\mathcal{D}=\left\{\mathcal{X} \in \mathbb{R}^{M}\right.$ : $\left|x_{i}-x_{j}\right|>d \forall i, j \in\{0, . ., M-1\}$ and $\left.i \neq j\right\}$, and $d$ depends on the size of the constellation and on the SNR. Since we are interested in finding non-degenerated constellation, we restrict the optimization process to $\mathcal{D}$. Then, a simplex method is used to perform the optimization with initial value in $\mathcal{D}$.

The alternative maximization method can at least increase the objective function in each iteration. In the experiments, we have observed that this method converges at least to a local maximum (denoted $\left.p_{i, j}^{*}(s), x_{j}^{*}(s), 0 \leq j \leq M-1,0 \leq i \leq|\mathcal{U}|-1\right)$. We discuss now the choice of $s$. Since we do not know a priori which value of $s$ may correspond to the satisfaction of the equality power constraint, we propose to use an iterative process as follows:

$$
s^{(k+1)}=\left[s^{(k)}-\gamma \cdot\left(P-\sum_{i=0}^{|\mathcal{U}|-1} \sum_{j=0}^{M-1} p_{i j}^{*}\left(s^{(k)}\right) \cdot\left(x_{j}^{*}\left(s^{(k)}\right)\right)^{2}\right)\right]^{+},
$$

where [.] $]^{+}$is defined as [.] $]^{+}=\max (., 0)$. The value of $s$ is increased or decreased with the sign of $P$ $\sum_{i=0}^{|\mathcal{U}|-1} \sum_{j=0}^{M-1} p_{i j}^{*}\left(s^{(k)}\right) \cdot\left(x_{j}^{*}\left(s^{(k)}\right)\right)^{2}$. The process stops when the power constraint is fulfilled. The proposed algorithm is summarized in Table 2. Obviously, when constellation symbols are constrained to the values of a standard constellation, $(P 2)$ which is defined in Table 2 will not be used. Similarly, when $P_{U X}$ is uniform, $(P 1)$ is not used. An alternative interpretation of this algorithm is to recognize that $L\left(P_{U X}, x_{0}, . ., x_{M-1}, s\right)$ is the Lagrangian dual of problem 9. Equations (17) and (18) are an iterative method for solving

$$
f(s)=\max _{P_{U X}, x_{0}, . ., x_{M-1}} L\left(P_{U X}, x_{0}, . ., x_{M-1}, s\right) .
$$

The dual optimization problem $\min _{\text {s.t. } s \geq 0} f(s)$ is solved in (19) with a gradient-type algorithm. Since $f(s)$ is convex [31], a gradient search method is guaranteed to converge to a global optimum.

\section{Result analysis}

\subsection{Point to point channel}

We present in this section the results of maximizing achievable rates for PtP case using $M$-PAM constellations with $M=4,8,16$ and for different values of SNR. To evaluate the contribution of constellation shaping, we compare, for a fixed SNR, the maximal achievable rate calculated by the algorithm proposed in the previous section to the 'standard constellation' rate, whose symbols are used with equal probability, at the same SNR in terms of SNR saving (called SNR shaping gain). The SNR shaping gain depicted in (Figure 4) is the gain obtained with a fully optimized constellation $\left(P_{\mathcal{X}}\right.$ and $\left.\mathcal{X}\right)$ compared to the standard $M$-PAM constellation and when symbols are used with the same probability. To avoid the complexity of constructing nearly optimal input distribution codes, another method for doing constellation shaping is to optimize only the position of symbols in the constellation. Each signal point is assumed to be chosen with the same probability; however, the position of each point in the constellation is optimized. The corresponding shaping gain is

\begin{tabular}{|c|c|c|}
\hline Step & & Solution \\
\hline Step 0 & & $s \leftarrow s^{(0)}$ \\
\hline \multirow[t]{4}{*}{ Step $k$} & Step 0 & $\mathcal{X} \leftarrow \mathcal{X}^{(0)}$ where $\mathcal{X}=\left(x_{0}, x_{1}, \ldots, x_{M-1}\right)$ \\
\hline & Step $\ell$ & $\begin{aligned} P_{U X}^{(\ell)} & =\arg \max _{P_{U X} \in \mathcal{C}} L\left(P U X, \mathcal{X}^{(\ell-1)}, s^{(k-1)}\right) \\
\mathcal{X}^{(\ell)} & =\arg \max \mathcal{X} L\left(P_{U X}^{(\ell)}, \mathcal{X}, s^{(k-1)}\right)\end{aligned}$ \\
\hline & $\begin{array}{l}\text { Stopping } \\
\text { criterion }\end{array}$ & $\left|L\left(P_{U X}^{(\ell)}, \mathcal{X}^{(\ell)}, s^{(k)}\right)-L\left(P_{U X}^{(\ell-1)}, \mathcal{X}^{(\ell-1)}, s^{(k-1)}\right)\right| \leq \epsilon L$ \\
\hline & & $\begin{array}{c}s^{(k)}=\left[s^{(k-1)}-\beta\left(P-\sum_{i, j} p_{i j}^{*}\left(s^{(k-1)}\right) \cdot\left(x_{j}^{*}\left(s^{(k-1)}\right)\right)^{2}\right)\right]^{+} \\
\text {where [.] }]^{+}=\max (., 0)\end{array}$ \\
\hline
\end{tabular}
given in (Figure 5). We observe the following: the shaping

\section{Table 2 Numerical solution for solving (9)}

Stopping

criterion

$\left|s^{(k)}-s^{(k-1)}\right| \leq \epsilon_{s}$ 


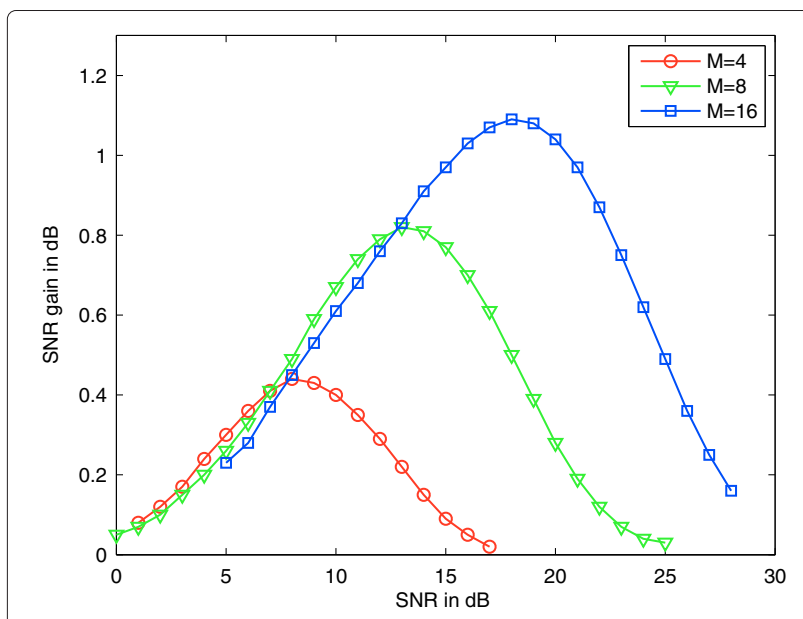

Figure 4 SNR shaping gain in $\mathrm{dB}$ for PtP channel. $\mathcal{X}$ and $P_{\mathcal{X}}$ are optimized.

gain depends on the SNR and on the size of the constellation. The maximum gain is obtained for mid-range SNR. The distribution of probability $P_{\mathcal{X}}$ (not reported) is very similar to the sampling of a Gaussian distribution. With the half-optimized constellation ( $\mathcal{X}$ only), a significant degradation is observed for mid-range SNR compared to that for the fully optimized constellation. Hence, we can conclude that symbol pdf optimization is useless at low and high SNR, whereas the fully optimized constellation is efficient for mid-range SNR, in which case the gain increases with the size of the constellation.

\subsection{Broadcast channel}

Current broadcast systems are using two practical transmission schemes for sending information to users: orthogonal schemes in which the time and/or frequency is

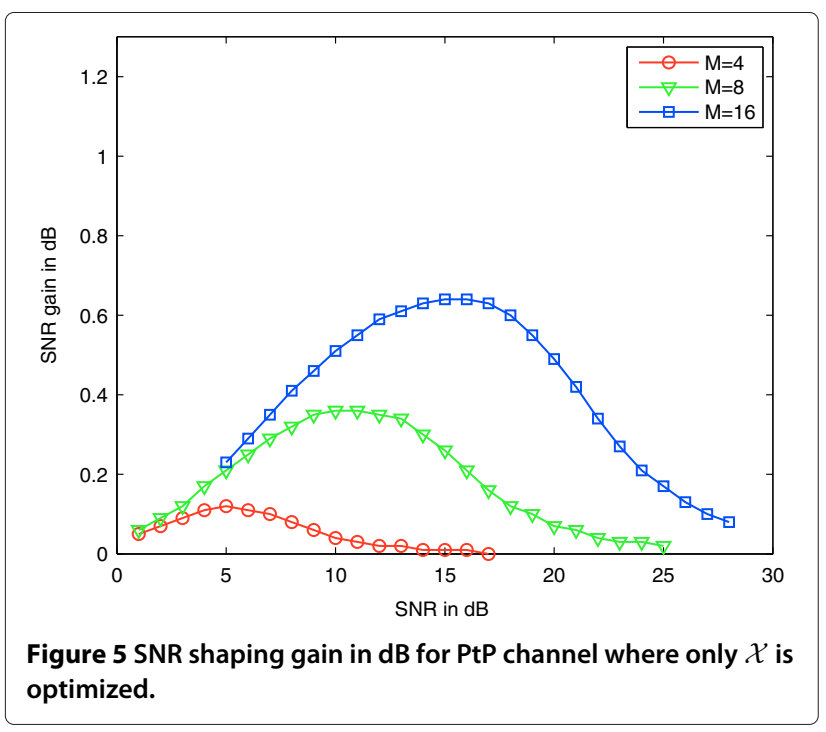

split between the users, and superposition modulation schemes where the constellation for each user is fixed. In this section, a comparison is provided between these standard schemes and various (more complex) transmission strategies such as superposition coding. The effect of constellation shaping is evaluated by analyzing the achievable rate region curves obtained for an $M$-PAM constellation $(M=4,8,16)$ and for several pairs $\left(S N R_{1}, S N R_{2}\right)$. The following schemes are considered:

- Time sharing using standard M-PAM (TS).

- $\mathrm{SM}$ - 3 possible configurations (see Table 1)

- $\mathrm{SC}-4$ possible configurations (see Table 1)

In the following, we denote by the 'case 1' of superposition modulation when $M_{1}=2, M_{2}=4$ and when $M_{1}=$ $2, M_{2}=8$. 'Case 2' is when $M_{1}=4, M_{2}=2$ and when $M_{1}=4, M_{2}=4$. 'Case 3' refers to the case when $M_{1}=$ $8, M_{2}=2$.

Achievable rate region curves are provided in Figures 6, $7,8,9,10$, and 11 for $M=4,8,16$. For each value of $M$, the display of the results is limited to two different pairs of SNR. In complement with the achievable rate region curves, comparisons are also conducted in terms of SNR savings for target achievable rates (maximum shaping gain) and in terms of maximum percentage of gain for user 1 . These two quantities are defined below.

Definition 1. Consider two transmission strategies $(A$ and $B)$. The pair of rates $\left(R_{1}+R_{2}, R_{2}\right)$ is achieved for $\left(\mathrm{SNR}_{1}, \mathrm{SNR}_{2}\right)$ with $A$ and for $\left(\mathrm{SNR}_{1}+\Delta \mathrm{SNR}, \mathrm{SNR}_{2}+\right.$ $\triangle \mathrm{SNR}$ ) with $B$. The shaping gain (with $A$ compared to $B$ ) is $\triangle \mathrm{SNR}$. The maximum shaping gain is defined as

$$
\mathrm{MG}_{\mathrm{SNR}_{d B}}(A \mid B)=\max _{R_{2}} \Delta \mathrm{SNR}
$$

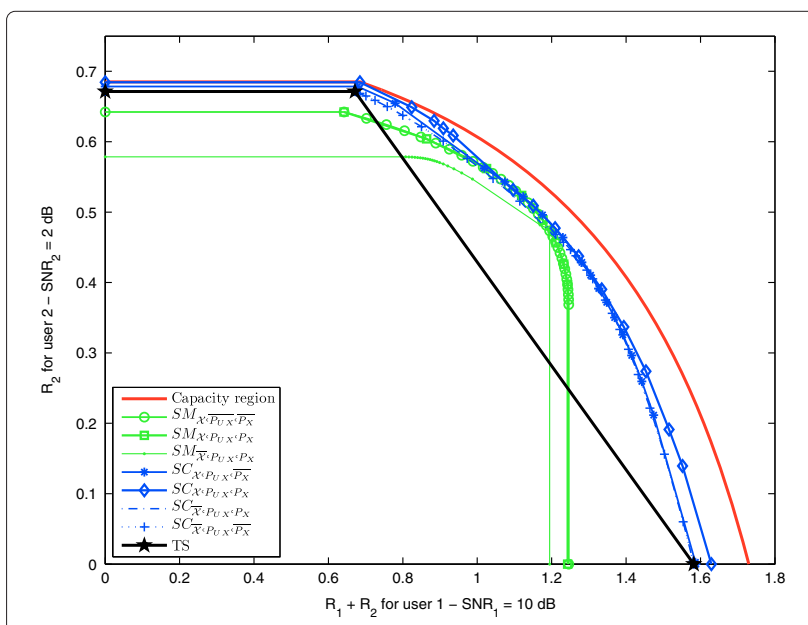

Figure 6 Achievable rate regions with $M=4$ and $\left(\mathrm{SNR}_{1}, \mathrm{SNR}_{2}\right)=$ (10 dB, $2 \mathrm{~dB})$. 


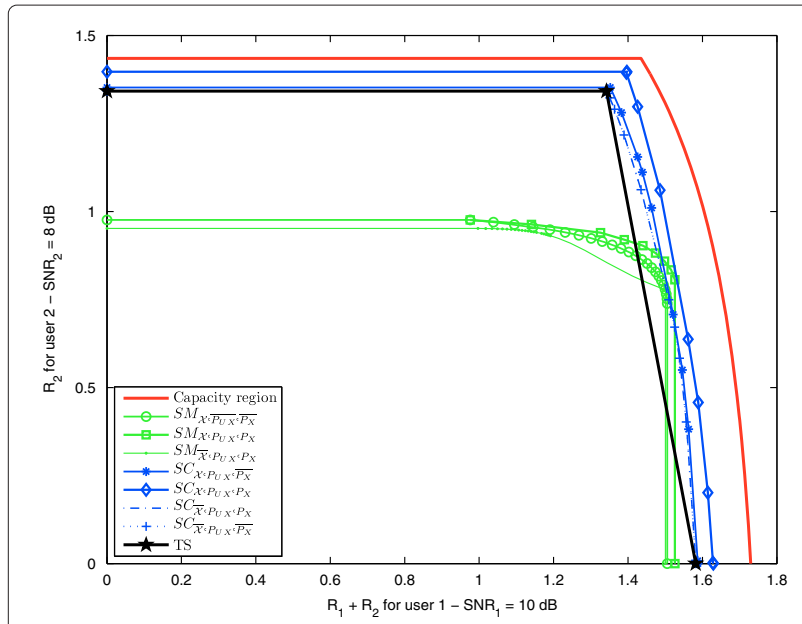

Figure 7 Achievable rate regions with $M=4$ and $\left(\mathrm{SNR}_{1}, \mathrm{SNR}_{2}\right)=$ (10 dB, $8 \mathrm{~dB})$.

Definition 2. Consider two transmission strategies $(A$ and $B)$. For a given pair of SNR $\left(\mathrm{SNR}_{1}, \mathrm{SNR}_{2}\right)$ and a fixed value of $R_{2}$, the achievable pair of rates is $\left(R_{1}^{A}+R_{2}, R_{2}\right)$ and $\left(R_{1}^{B}+R_{2}, R_{2}\right)$ with $A$ and $B$, respectively. The gain on the achievable rate for user 1 is given by

$$
G_{R_{1}}(A \mid B)=\frac{\left(R_{1}^{A}+R_{2}\right)-\left(R_{1}^{B}+R_{2}\right)}{R_{1}^{B}+R_{2}} \cdot 100(\%) .
$$

The maximum gain on the achievable rate for user 1 (with $A$ compared to $B$ ) is given by

$$
\mathrm{MG}_{R_{1}}(A \mid B)=\max _{R_{2}} G_{R_{1}}(A, B) .
$$

\subsubsection{Superposition modulation}

In this section, the three possible configurations of superposition modulation are compared. We can see from

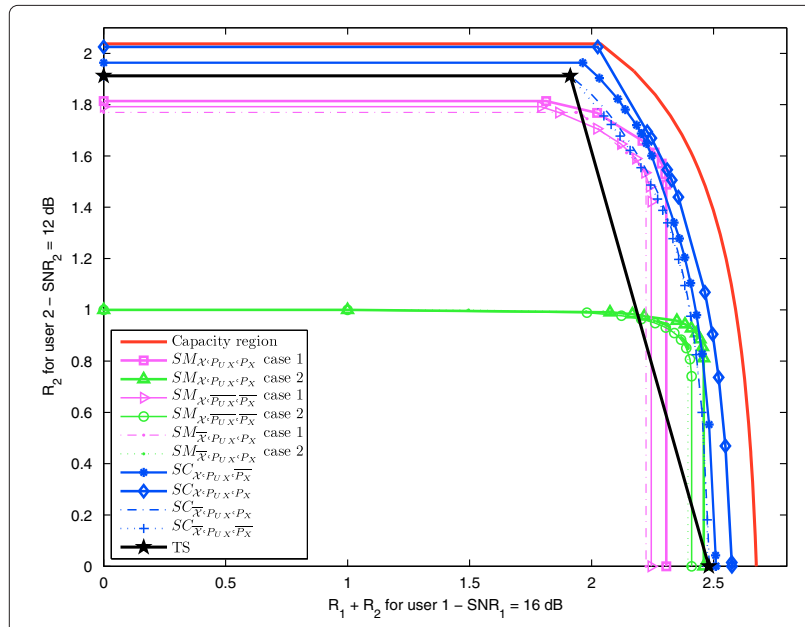

Figure 8 Achievable rate regions with $M=8$ and $\left(\mathrm{SNR}_{1}, \mathrm{SNR}_{2}\right)=$ $(16 \mathrm{~dB}, 12 \mathrm{~dB})$.

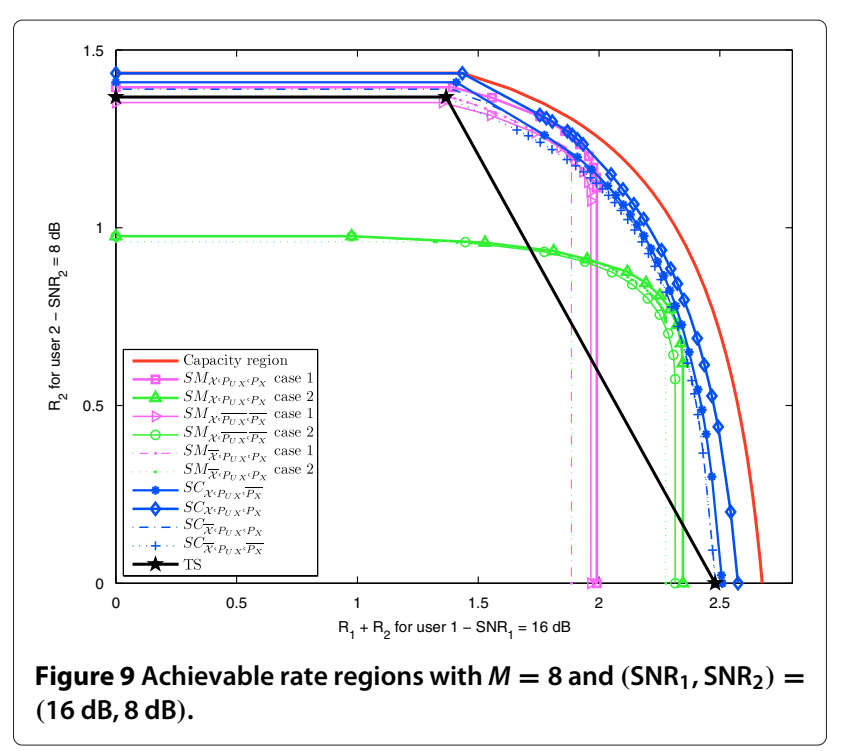

Figures $6,7,8,9,10$, and 11 that $\mathrm{SM}_{\mathcal{X}, \overline{P_{U X}}, \overline{P_{X}}}$ (optimization of $\mathcal{X}$ only) outperforms $\mathrm{SM}_{\overline{\mathcal{X}}, P_{U X}, P_{X}}$ (optimization of $P_{U X}$ only) in terms of maximal achievable rates per user when $M=4$. For $M=8$ and $16, \mathrm{SM}_{\overline{\mathcal{X}}, P_{U X}, P_{X}}$ can achieve slightly higher rates than $\mathrm{SM}_{\mathcal{X}, \overline{P_{U X}}, \overline{P_{X}}}$. The implementation of a system with constellation symbols with non-standard positions and generated with the same probability is less complex than the implementation of a system which generates symbols with non-uniform joint distribution of probability. Thus, $\mathrm{SM}_{\overline{\mathcal{X}}, P_{U X}, P_{X}}$ does not seem to be of interest since it is not very efficient in terms of achievable rates and is more complex to implement.

Figures of achievable rate region show that an improvement can be obtained with $\mathrm{SM}_{\mathcal{X}, P_{U X}, P_{X}}$ (full optimization) compared to $\mathrm{SM}_{\mathcal{X}, \overline{P_{U X}}, \overline{P_{X}}}$ (optimization of $\mathcal{X}$ only) and depending on $\delta_{\mathrm{SNR}}=\mathrm{SNR}_{1}-\mathrm{SNR}_{2}$. Numerical values of

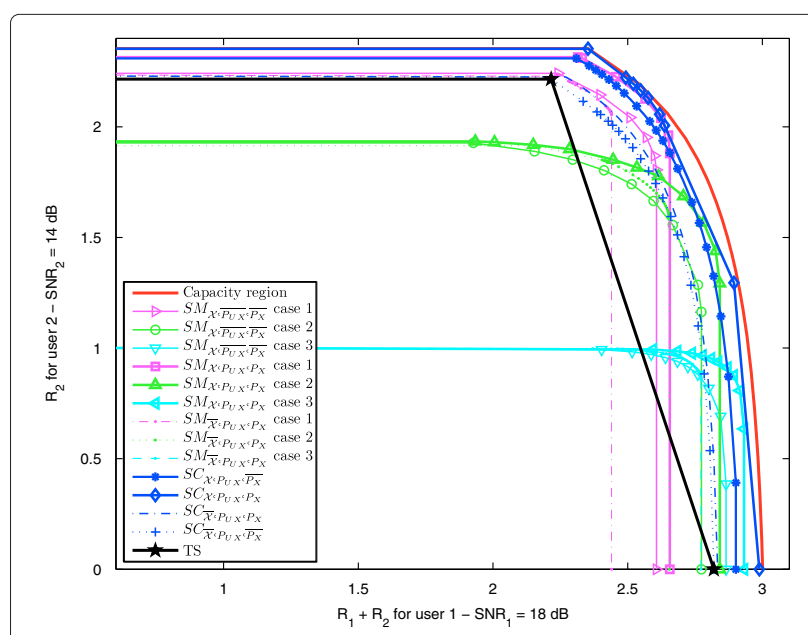

Figure 10 Achievable rate regions with $M=16$ and $\left(\mathrm{SNR}_{1}, \mathrm{SNR}_{2}\right)=$ $(18 \mathrm{~dB}, 14 \mathrm{~dB})$. 


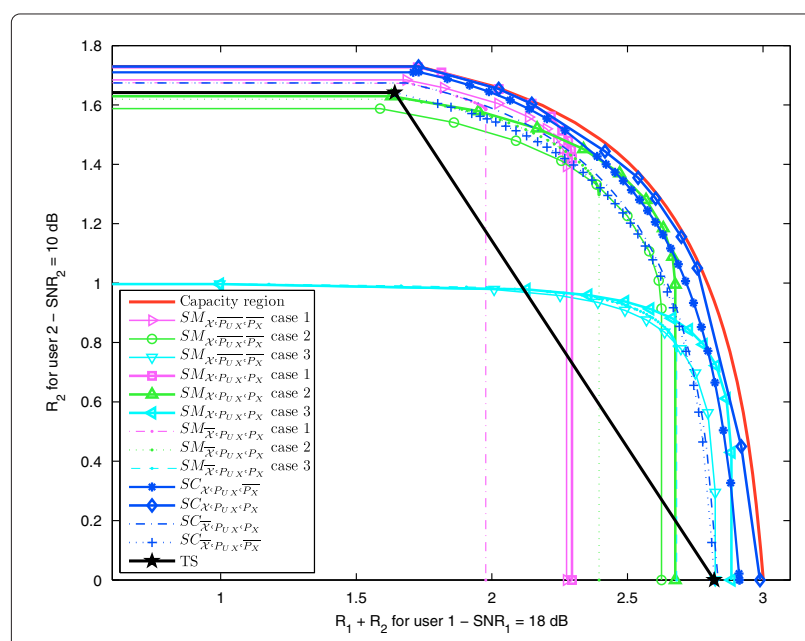

Figure 11 Achievable rate regions with $M=16$ and $\left(\mathrm{SNR}_{1}, \mathrm{SNR}_{2}\right)=$ $(18 \mathrm{~dB}, 10 \mathrm{~dB})$.

the maximum gain in achievable rate $\left(\mathrm{MG}_{R_{1}}\right)$ and of the maximum SNR savings $\left(\mathrm{MG}_{\mathrm{SNR}_{d B}}\right)$ are given in Table 3. We observe the following: a slight gain in terms of achievable rates can be translated into a noticeable gain in terms of SNR saving. The maximum shaping gain increases with the constellation size. Thus, constellation shaping for the SM strategy seems more useful for high values of $M$. The analysis of the optimal matrix $P_{U X}$ (results not reported) leads to the conclusion that $X_{1}$ and $X_{2}$ are not independent in general when using finite-size constellations. We observe also that the maximum shaping gain for $\mathrm{SM}_{\mathcal{X}, P_{U X}, P_{X}}$ versus $\mathrm{SM}_{\mathcal{X}, \overline{P_{U X}}, \overline{P_{X}}}$ increases when $\delta_{\mathrm{SNR}}$ decreases, independently of $M$. In particular, full optimization (vs. optimization of the symbol position) does not provide significant improvement for large SNR gap in the SM strategy.

\begin{tabular}{|c|c|c|c|c|}
\hline$M$ & $\mathrm{SNR}_{1}$ & $\mathrm{SNR}_{2}$ & $\mathrm{MG}_{\mathrm{SNR}_{d B}}(A \mid B)$ & $\mathrm{MG}_{R_{1}}(A \mid B)$ \\
\hline & & 8 & 0.39 & $7.46 \%$ \\
\hline \multirow[t]{4}{*}{4} & 10 & 6 & 0.17 & $3.51 \%$ \\
\hline & & 4 & 0.05 & $1.77 \%$ \\
\hline & & 2 & 0.01 & $0.38 \%$ \\
\hline & & 14 & $0.71\left(M_{1}=4, M_{2}=2\right)$ & $20.17 \%\left(M_{1}=4, M_{2}=2\right)$ \\
\hline \multirow[t]{4}{*}{8} & 16 & 12 & $0.57\left(M_{1}=4, M_{2}=2\right)$ & $13.21 \%\left(M_{1}=4, M_{2}=2\right)$ \\
\hline & & 10 & $0.41^{\left(M_{1}=4, M_{2}=2\right)}$ & $13.07 \%\left(M_{1}=2, M_{2}=4\right)$ \\
\hline & & 8 & $0.33^{\left(M_{1}=2, M_{2}=4\right)}$ & $18.93 \%\left(M_{1}=2, M_{2}=4\right)$ \\
\hline & & 16 & $1.05^{\left(M_{1}=8, M_{2}=2\right)}$ & $10.67 \%\left(M_{1}=8, M_{2}=2\right)$ \\
\hline \multirow[t]{3}{*}{16} & 18 & 14 & $0.87^{\left(M_{1}=8, M_{2}=2\right)}$ & $11.54 \%\left(M_{1}=8, M_{2}=2\right)$ \\
\hline & & 12 & $0.64\left(M_{1}=8, M_{2}=2\right)$ & $12.08 \%\left(M_{1}=4, M_{2}=4\right)$ \\
\hline & & 10 & $0.49^{\left(M_{1}=8, M_{2}=2\right)}$ & $19.53 \%\left(M_{1}=4, M_{2}=4\right)$ \\
\hline
\end{tabular}

\subsubsection{Time sharing or superposition modulation?}

This section compares two strategies (TS and SM) classically considered in broadcast systems. In Figures 6 and $7(M=4)$, we observe that the achievable rate region can be split into two parts. Indeed, for small and large values of $R_{2}$, TS is better than SM. On the contrary, SM is better than TS for middle-range values of $R_{2}$. Under a given rate requirement for one user, we can thus determine the best transmission strategy. We can also observe that the region in which SM is better than TS becomes small for larger values of $\mathrm{SNR}_{2}$. With $M=8$ (Figures 8 and 9), the area in which SM is better than TS increases (compared to $M=4$ ) by considering the union of the two possible configurations for SM: $M_{1}=2, M_{2}=4$ (case 1) and $M_{1}=4, M_{2}=2$ (case 2). This is particularly true when $\delta_{\mathrm{SNR}}$ increases. We also observe that TS can achieve higher rates than SM (case 1) for good $\mathrm{SNR}_{2}$ values. Indeed, the maximum rate of user 2 with $S M$ is the maximum individual rate for a 4-PAM constellation, whereas it is the individual user rate that achieved using standard 8-PAM in the TS case. For low $\mathrm{SNR}_{2}$ values, optimized 4PAM may achieve higher rate than standard 8-PAM; thus, SM becomes better in this interval. For a 16-PAM constellation (Figures 10 and 11), SM is always better than TS for the studied pairs of $\left(\mathrm{SNR}_{1}, \mathrm{SNR}_{2}\right)$. Table 4 shows the maximum percentage of improvement in achievable rate of user 1 by TS when using $\mathrm{SM}_{\mathcal{X}, P_{U X}, P_{X}}$ (full optimization) strategy in the interval, where $\mathrm{SM}_{\mathcal{X}, P_{U X}, P_{X}}$ is better than TS. Clearly, the maximum percentage of improvement increases when $\delta_{\mathrm{SNR}}$ increases, and an important gain is obtained for high values of $\delta_{\mathrm{SNR}}$ as in the case of $\mathrm{SNR}_{1}=\delta_{\mathrm{SNR}}=10 \mathrm{~dB}$ for a 4 -PAM, where the percentage of gain on achievable rate of user 1 varies between $0 \%$ and $40.7 \%$. For a 8 -PAM constellation, the percentage of gain

Table 4 Comparison of $S M_{\mathcal{X}, P_{U X}, P_{X}}(A)$ vs. TS (B) and comparison of $S C_{\mathcal{X}, P_{U X}, P_{X}}(A)$ vs. TS $\cup S M_{\mathcal{X}, P_{U X}, P_{X}}(C)$

\begin{tabular}{|c|c|c|c|c|}
\hline$M$ & $\mathrm{SNR}_{1}$ & $\mathrm{SNR}_{2}$ & $\mathrm{MG}_{R_{1}}(A \mid B)$ & $\mathrm{MG}_{R_{1}}(A \mid C)$ \\
\hline & & 8 & $6.13 \%$ & $6.72 \%$ \\
\hline \multirow[t]{5}{*}{4} & & 6 & $11.14 \%$ & $11.65 \%$ \\
\hline & 10 & 4 & $18.50 \%$ & $16.69 \%$ \\
\hline & & 2 & $28.43 \%$ & $18.9 \%$ \\
\hline & & 0 & $40.70 \%$ & $23.54 \%$ \\
\hline & & 14 & $7.80 \%\left(M_{1}=2, M_{2}=4\right)$ & $7.89 \%$ \\
\hline \multirow[t]{4}{*}{8} & 16 & 12 & $13.60 \%\left(M_{1}=2, M_{2}=4\right)$ & $11.43 \%$ \\
\hline & & 10 & $21.15 \%\left(M_{1}=2, M_{2}=4\right)$ & $14.96 \%$ \\
\hline & & 8 & $30.21 \%\left(M_{1}=2, M_{2}=4\right)$ & $14.71 \%$ \\
\hline & & 16 & $10.36 \%\left(M_{1}=2, M_{2}=8\right)$ & $2.96 \%$ \\
\hline \multirow[t]{3}{*}{16} & 18 & 14 & $16.42 \%\left(M_{1}=4, M_{2}=4\right)$ & $2.94 \%$ \\
\hline & & 12 & $24.68 \%\left(M_{1}=4, M_{2}=4\right)$ & $5.29 \%$ \\
\hline & & 10 & $35.08 \%\left(M_{1}=4, M_{2}=4\right)$ & $4.80 \%$ \\
\hline
\end{tabular}


on achievable rate of user 1 varies between $0 \%$ and $30.21 \%$ when $\mathrm{SNR}_{1}=16 \mathrm{~dB}$ and $\delta_{\mathrm{SNR}}=8 \mathrm{~dB}$. For a $16-\mathrm{PAM}$, the percentages of improvements can be up to $35.08 \%$ when $\mathrm{SNR}_{1}=18 \mathrm{~dB}$ and $\delta_{\mathrm{SNR}}=8 \mathrm{~dB}$. We can conclude that $\mathrm{SM}$ is a better option than TS especially for large $\delta_{\mathrm{SNR}}$ values. TS is optimal in the region, where we want to maximize the rate of user 2 for good values of $\mathrm{SNR}_{2}$ because the single user rate achieved by TS is the rate achieved using standard $M$-PAM constellation (the constellation is split between users with SM). Thus, SM seems more gainful than TS when we want to serve users with very diverse SNRs.

\subsubsection{Is superposition coding necessary?}

For the three constellations under consideration $(M=$ $4,8,16)$, the maximal achievable rate region obtained by the optimal general case of superposition coding when we consider the general form of $P_{U X}(\mathrm{SC})$ can achieve, depending on $M$ and user SNRs, a large region of rate pairs $\left(R_{1}+R_{2}, R_{2}\right)$ that cannot be achieved neither by TS nor

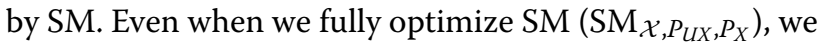
are far from maximal achievable rate region. Sometimes, the maximal achievable rate region curve is very close or even coincides with the $\mathrm{SM}_{\mathcal{X}, P_{U X}, P_{X}}$ achievable rate region in a pair of rates $\left(R_{1}^{*}+R_{2}^{*}, R_{2}^{*}\right)$. This is the case when $\mathrm{SM}_{\mathcal{X}, P_{U X}, P_{X}}$ is the optimal superposition coding in terms of achievable rates. We can see for example in Figure 6 that the pair of rates $\left(R_{1}^{*}+R_{2}^{*}=1.096, R_{2}^{*}=0.531\right.$ which corresponds to the optimal rate pair when we optimize the general case of SC for $\theta=0.23$ ) is an intersection point with $\mathrm{SM}_{\mathcal{X}, P_{U X}, P_{X}}$ achievable rate region.

We are interested now in the numerical evaluation of the gain in rate of user $1\left(R_{1}+R_{2}\right)$ when we use $\mathrm{SC}_{\mathcal{X}, P_{U X}, P_{X}}$ (full optimization) compared to the best strategy between TS and SM. This gain $\left(\mathrm{MG}_{R_{1}}\left(\mathrm{SC}_{\mathcal{X}, P_{U X}, P_{X}} \mid T S \bigcup \mathrm{SM}_{\left.\mathcal{X}, P_{U X}, P_{X}\right)}\right.\right.$ calculated in $\%$ is the distance between the limit of the maximal achievable rate region and the limit of the union of achievable rate regions of TS and $\mathrm{SM}_{\mathcal{X}, P_{U X}, P_{X}}$.

The results are reported in Table 4 . We observe that the part of the maximal achievable rate region which is unachievable by TS and SM is bigger when $M$ is small because we observe that for the case of 4-PAM, we have one configuration for SM. However, we have two configurations of SM for 8-PAM constellation and three configurations for 16-PAM constellation. Thus, when $M$ increases, the union of achievable rates for all SM cases tends to the sets of achievable rates by the general superposition coding. Asymptotically, we know that when $M \rightarrow \infty, \mathrm{SM}_{\mathcal{X}, P_{U X}, P_{X}}$ is the optimal superposition coding scheme because it allows the capacity region for two-user AWGN BC using Gaussian alphabet for each user to be achieved. Thus, the maximum gain in user 1 rate decreases when the constellation order $M$ increases. We observe also that the gain in achievable rates is high for high values of $\delta_{\mathrm{SNR}}$. On the other hand, the experiments show that by using the general superposition coding strategy with the constraint that symbols should be equiprobable $\left(\mathrm{SC}_{\mathcal{X}, P_{U X}, \overline{P_{X}}}\right)$, the loss is limited compared to the full optimization $\left(\mathrm{SC}_{\mathcal{X}, P_{U X}, P_{X}}\right), 4.84 \%, 7.66 \%$, and $3.94 \%$ for the simulated pairs of $\left(\mathrm{SNR}_{1}, \mathrm{SNR}_{2}\right)$ when $M=4,8$, and 16 , respectively. This means that we can use equiprobable symbols with, in general, a small loss in achievable rates. However, $\mathrm{SC}_{\mathcal{X}, P_{U X}, \overline{P_{X}}}$ is not an interesting case when $\mathrm{SM}_{\mathcal{X}, P_{U X}, P_{X}}$ can achieve better rates since SM is less complex to implement than SC.

Moreover, with standard $M$-PAM symbols, the two possible configurations $\left(\mathrm{SC}_{\overline{\mathcal{X}}, P_{U X}, P_{X}}\right.$ (optimization of $P_{U X}$ and $P_{X}$ ) and $\mathrm{SC}_{\overline{\mathcal{X}}, P_{U X}, \overline{P_{X}}}$ (optimization of $P_{U X}$ only)) give very similar results in most considered pairs of SNR. We also observe that the loss in maximum achievable rate experienced by user 1 with $\mathrm{SC}_{\overline{\mathcal{X}}, P_{U X}, P_{X}}$ is less than $10 \%$ under the rate experienced with

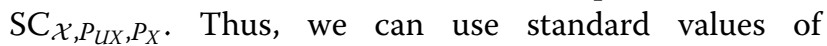
symbol positions without losing much on achievable rates.

In general, one can conclude that fixing constellations of users (i.e., assigning labels to the constellation so that we distinguish between the bits intended for each user) is not optimal for coding and may result in important loss in terms of rates for systems using finitesize constellations especially for low-order constellations. A better solution is to determine the optimal alphabet of the auxiliary alphabet $U$ which is not necessarily a constellation and then to generate the codewords $x^{n}$ which are not necessarily the sum of two codewords (see Section 3.4).

\section{Application: coverage extension}

We first consider a transmission over a broadcast channel with finite size input alphabet. For simplicity of the illustration and without loss of generality, let us assume that the existing user alphabet belongs initially to a standard constellation whose symbols are used with equal probability. We assume that the existing user is at distance $d_{0}$ from the sender achieving a rate $R_{0}$. Some information is also to be transmitted to an upgraded layer of users. The sender can use up to 16 symbols, then several transmission schemes can be used. We are interested in comparing the transmission schemes to serve the new user under two scenarios: either the new user is closer to the transmitter than the existing user or the new user is farther than the existing one. For a target rate $R_{0}$ that is fixed for the existing user and achievable using a standard $M$-PAM and equiprobable symbols, we are interested in determining the variation of the coverage's diameter ratio between the two layer of users as a 
function of the achievable rate by the upgraded user for various broadcast transmission strategies. We assume that $\mathrm{SNR} \propto \frac{1}{d^{2}}$.

\subsection{The sender can use up to 16 symbols 6.1.1 Scenario 1}

In this scenario, the system consists initially of one layer of users. Now, assume that the data information is also to be transmitted to a second layer of users with higher SNR. In the following, we keep the notation from the preceding section, where the user with greater SNR is denoted by user 1 . Thus, in this scenario, the legacy receivers are denoted by user 2 which is at a distance $d_{2}$ from the transmitter and achieving a rate $R_{0}$ when the data is modulated using standard 4-PAM constellation and equiprobable symbols. The upgraded receivers are denoted by user 1 $\left(\mathrm{SNR}_{1}>\mathrm{SNR}_{2}\right)$. We intend that the good user receives more throughput than user 2 via the use of 16-PAM.

In this example $\mathrm{SNR}_{2}$ is fixed to $10 \mathrm{~dB}$. Initially, user 2's alphabet belongs to a 4-PAM standard constellation (see section 3.1), and the rate transmitted to user 2 is $R_{0}=1.582 \mathrm{bits} / \mathrm{ch}$. use.

Now, a new layer of users called user 1 is introduced in the system with $\mathrm{SNR}_{1}>\mathrm{SNR}_{2}$. Our target is to provide the maximum bit rate to the new user without changing $R_{0}$ or $d_{0}$ and using a 16-PAM. By enlarging the constellation and optimizing the symbol positions and probability distribution, we ensure that the rate of the initial user will not decrease after introducing a new user.

Consider now the results for the following strategies which can achieve a positive private-message rate for user

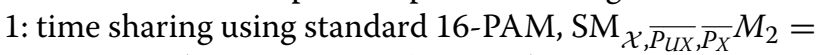
$8 / M_{1}=2$ (optimization of $\mathcal{X}$ only), $\mathrm{SM}_{\mathcal{X}, P_{U X}, P_{X} M_{2}=}$ $8 / M_{1}=2$ (full optimization) and $\mathrm{SC}_{\mathcal{X}, P_{U X}, P_{X}}$ (full optimization). Figure 12 illustrates the variation of $d_{1} / d_{2}$,

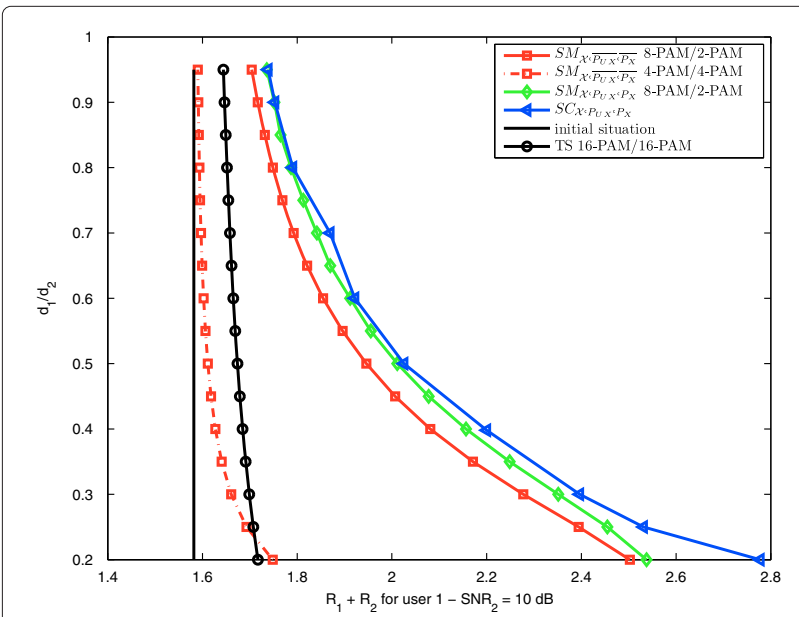

Figure 12 Coverage ratio $d_{1} / d_{2}$ as function of the achievable rate for user 1 . which is the ratio of the diameter of the coverage area for user 1 over the diameter of the initial coverage area for user 2 , as a function of the achievable rate for user 1 for a target rate $R_{0}=1.582$ for user 2 .

Let us assume for example that the new user is midway between the transmitter and user $2\left(d_{1} / d_{2}=0.5\right)$. Figure 12 shows that the most simple case of superposition modulation $\left(\mathrm{SM}_{\mathcal{X}, \overline{P_{U X}}, \overline{P_{X}}} M_{2}=8 / M_{1}=2\right)$ provides $16.3 \%$ more bit rate than time sharing for the new user. If we move immediately to a more complex case and opti-

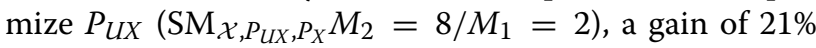
is obtained on the bit rate of user 1 compared to time sharing. This gain on achievable rate for the new user is equivalent to a gain of $1 \mathrm{~dB}$ on $\mathrm{SNR}_{1}$ compared to superposition modulation with uniform $P_{U X}$. However, if we move to the most general case of superposition coding, it does not provide significant gain compared to superposition modulation.

Now, we assume that the new user is close to the transmitter such that $d_{1} / d_{2}=0.2$. We observe that the gain on the bit rate of user 1 using the simple case of superposition modulation increases to $45.7 \%$ compared to time sharing. By moving to a more complex case $\left(\mathrm{SM}_{\mathcal{X}, P_{U X}, P_{X}} M_{2}=8 / M_{1}=2\right)$, a gain of $47.8 \%$ is obtained on the bit rate of user 1 compared to time sharing. We observe also that it is relevant in this case to move to the most general case of superposition coding since it provides a gain of $61.8 \%$ on the bit rate of user 1 compared to time sharing.

Consequently, using superposition modulation provides always noticeable gain compared to time sharing. The general case of superposition coding $\mathrm{SC}_{\mathcal{X}, P_{U X}, P_{X}}$ is useful when user 1 is close to the transmitter, but not when it is close to user 2 .

\subsubsection{Scenario 2}

Initially, consider a system of one layer of users, denoted by user 1 , at a distance $d_{1}$ from the transmitter and achieving a rate $R_{0}$. Moreover, the alphabet of user 1 belongs to a standard 8-PAM constellation. In this example, $\mathrm{SNR}_{1}$ is fixed to $18 \mathrm{~dB}$. Thus, user 1 can achieve a rate $R_{0}=$ $2.73 \mathrm{bits} / \mathrm{ch}$. use in the initial situation. In this scenario, we want to serve a second layer of users denoted by user 2 which is farther to the transmitter than the existing user, i.e., $\mathrm{SNR}_{2}<\mathrm{SNR}_{1}$.

Achievable rates for user 2 are obtained at different distance $d_{2}$ from the transmitter and using various transmission strategies for a target rate of user 1 equal to $R_{0}$ and a coverage diameter for user 1 fixed to $d_{1}$. Figure 13 illustrates the variation of $d_{2} / d_{1}$, which is the ratio of the diameter of the coverage area for user 2 over the diameter of the initial coverage area for user 1 , as a function of the achievable rate for user 2 when a target rate for user 1 is fixed to $R_{0}=2.73 \mathrm{bits} / \mathrm{ch}$. use. 


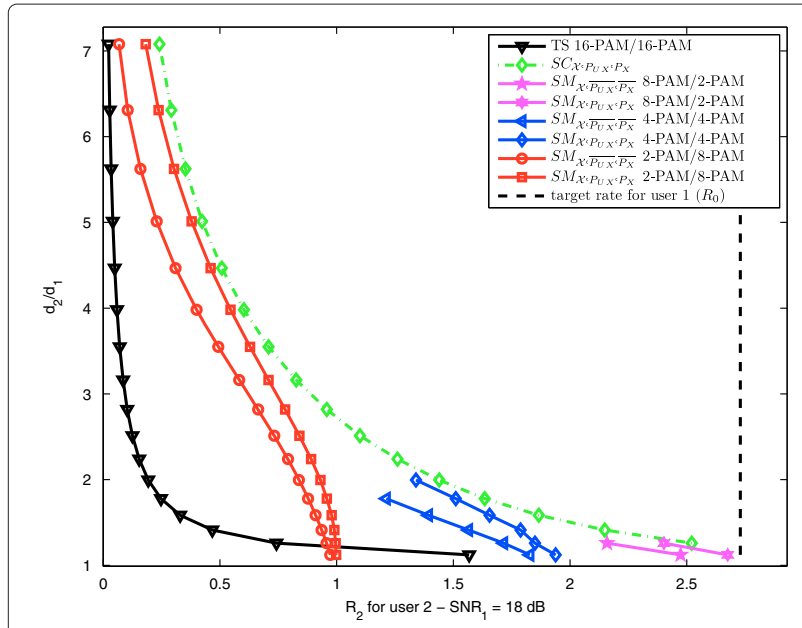

Figure 13 Coverage ratio $d_{2} / d_{1}$ as function of the achievable rate for user 2 .

We observe in Figure 13 that superposition modulation can always achieve better rates for user 2 than time sharing using 16-PAM. Let us assume first that we want to increase the diameter of the coverage area for the new user (user 2) such that $d_{2} / d_{1}=4$. Time sharing provides a bit rate less than $0.06 \mathrm{bits} / \mathrm{ch}$. use. The most simple case

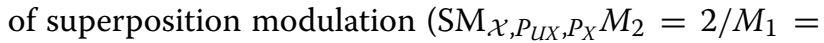
8) provides a significant improvement on the achievable rate for user 2 which is equal to $0.4 \mathrm{bits} / \mathrm{ch}$. use in this case. If we increase the complexity by optimizing the joint probability distribution $P_{U X}$, we obtain $35 \%$ more bit rate

Table 5 Comparison of $S C_{\mathcal{X}, P_{U X}, P_{X}}$ and $S M_{\mathcal{X}, P_{U X}, P_{X}} M_{2}-\mathrm{PAM} / M_{1}-\mathrm{PAM}$ w.r.t $\mathrm{MG}_{R_{2}}(\%)$

\begin{tabular}{lccc}
\hline $\boldsymbol{d}_{\mathbf{2}} / \boldsymbol{d}_{\mathbf{1}}$ & $\mathbf{S N R}_{\mathbf{2}}$ & $\mathbf{M G}_{\boldsymbol{R}_{\mathbf{2}}}$ & $\boldsymbol{M}_{\mathbf{2}} / \boldsymbol{M}_{\mathbf{1}}$ \\
\hline 1.2589 & 16 & 4.9416 & $8 / 2$ \\
1.4125 & 15 & 20.1521 & $4 / 4$ \\
1.5849 & 14 & 12.7522 & $4 / 4$ \\
1.7783 & 13 & 8.2192 & $4 / 4$ \\
1.9953 & 12 & 7.4536 & $4 / 4$ \\
2.2387 & 11 & 41.4993 & $2 / 8$ \\
2.5119 & 10 & 30.8293 & $2 / 8$ \\
2.8184 & 9 & 22.9121 & $2 / 8$ \\
3.1623 & 8 & 16.7443 & $2 / 8$ \\
3.5481 & 7 & 12.6033 & $2 / 8$ \\
3.9811 & 6 & 10.5427 & $2 / 8$ \\
4.4668 & 5 & 10.3343 & $2 / 8$ \\
5.0119 & 4 & 11.7414 & $2 / 8$ \\
5.6234 & 3 & 16.0961 & $2 / 8$ \\
6.3096 & 2 & 22.8535 & $2 / 8$ \\
7.0795 & 1 & 32.6194 & $2 / 8$ \\
\hline
\end{tabular}

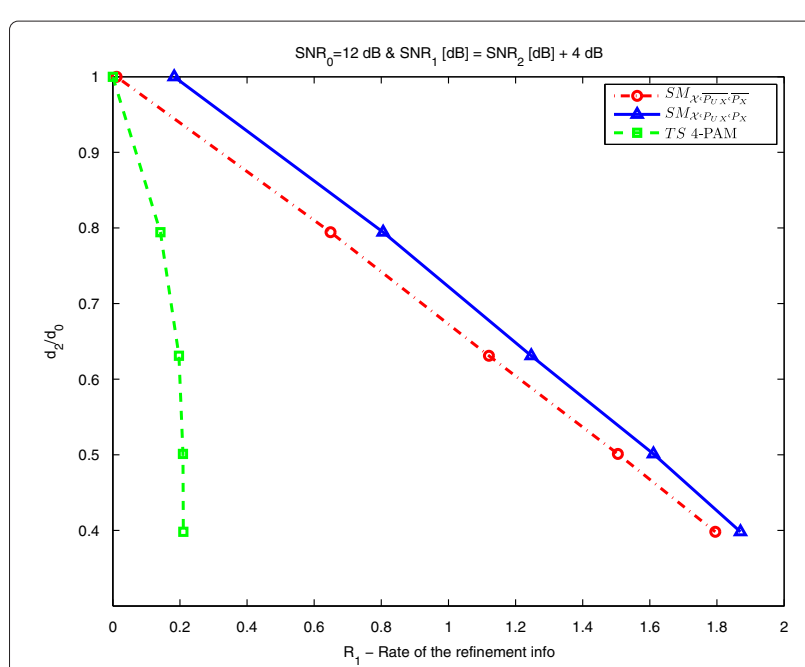

Figure 14 Reduction in legacy coverage $d_{2} / d_{0}$ in function of the rate of the refinement $R_{1}$.

for user 2 comparing to superposition modulation with uniform $P_{U X}$. If we move to the general case of superposition coding, we gain only $10 \%$ on the bit rate of the new user compared to superposition modulation (see Table 5). However, when the new layer of users is at distance $d_{2}=2.25 d_{1}$, the general case of superposition coding provides a significant gain of $41 \%$ on the achievable rate of user 2 comparing to superposition modulation.

Consequently, the general case of superposition coding can bring significant gains compared to superposition modulation, depending on the diameter of the coverage area for the new layer of users. For superposition modulation, optimizing the joint distribution of probability $P_{U X}$ provides often significant shaping gains.

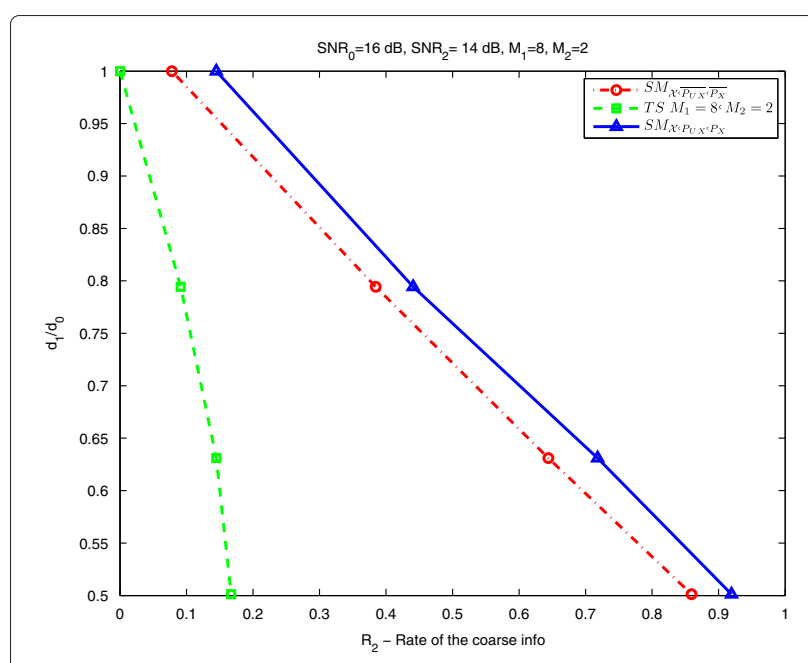

Figure 15 Reduction in legacy coverage $d_{1} / d_{0}$ in function of the rate of the coarse information $R_{2}$. 


\subsection{The cardinality of the existing user alphabet is kept fixed :}

In this section, we study scenario 1 (and 2) supposing that the legacy receivers will continue working as in the initial situation, still using 4-PAM (8-PAM). The system consists initially one layer of users at distance $d_{0}$ from the transmitter and achieves a rate $R_{0}$. Now, we want to change the transmitter, such that the upgraded receivers closer (farther) in range will be able to decode a refinement (coarse) layer and use a 16-PAM constellation. Thus, only time sharing with $M_{1}=M_{2}=4\left(M_{1}=8, M_{2}=2\right)$ and superposition modulation strategies can be used. We aim to study how small the reduction in legacy coverage can be made, depending on the rate of the refinement (coarse) information achieved by the upgraded users. Thus, suppose that the legacy coverage can be reduced from $d_{0}$ to $d_{2}$ (from $d_{0}$ to $d_{1}$ ). We have studied this problem for $\mathrm{SNR}_{0}=12 \mathrm{~dB}$ and for $\mathrm{SNR}_{1}-\mathrm{SNR}_{2}=4 \mathrm{~dB}$ in scenario 1 (and for $\mathrm{SNR}_{0}=16$ and $\mathrm{SNR}_{2}=14 \mathrm{~dB}$ in scenario 2). Figures 14 and 15 represent the reduction in coverage $d_{2} / d_{0}$ (and $d_{1} / d_{0}$ respectively) as a function of the rate of the refinement $R_{1}$ (of the coarse $R_{2}$ ), while the rate achieved by the legacy receivers is kept fixed to its initial situation, i.e., $R_{0}$.

We observe in Figures 14 and 15 that the gain of superposition modulation strategies over time sharing becomes more important when $d_{2} / d_{0}\left(d_{1} / d_{0}\right)$ is small. These figures show that using superposition modulation when both symbol positions and $P_{U X}$ are optimized, we gain around $5 \%$ from the initial coverage compared to the case of superposition modulation where symbols are used with equal probability. We can observe also that a reduction of only $10 \%$ and $20 \%$ in coverage area for the existing user can serve the upgraded user with a rate up to $20 \%$ and $35 \%$ (9\% and 15\%) from the rate achieved by the legacy users, using $\mathrm{SM}_{\mathcal{X}, \overline{P_{U X}}, \overline{P_{X}}}$. Consequently, by using $\mathrm{SM}_{\mathcal{X}, \overline{P_{U X}}, \overline{P_{X}}}$, the legacy receivers still use 4-PAM (8-PAM in scenario 2), and we can serve a new layer of users with an acceptable rate, a small reduction in coverage area, and with less complexity compared to $\mathrm{SM}_{\mathcal{X}, P_{U X}, P_{X}}$.

\section{Conclusion}

In this work we considered the problem of maximizing the achievable rate region for power-constrained AWGN broadcast channel of two users using $M$-PAM constellations. The achievable rate region is given for various transmission strategies. Maximal achievable rate region for superposition coding and superposition modulation is obtained using constellation shaping. An iterative algorithm was proposed to solve this optimization problem. Then, the efficiency of several strategies are compared. For superposition modulation, the results showed that constellation shaping seems more useful for high values of $M$. Moreover, the gain in using a complex case of superposition modulation increases when the SNR gap between users decreases. We observed also that superposition modulation outperforms time sharing in a large part of the achievable rate region. On the other hand, it is shown that using the general case of superposition coding can bring important gains compared to classical schemes. We observed also that in the case of finite input alphabet, superposition modulation is not the optimal strategy as in the case of Gaussian input alphabets. Finally, in order to make clear that this paper provides useful tools for the system designer, we considered two scenarios of coverage areas and user alphabets where the systems served initially one layer of users. Then, we propose to serve a second layer of users, and we evaluate the achievable rate of the new layer depending on the broadcast strategy. To improve the system performance compared to time sharing, we can optimize the joint probability distribution and symbol positions of the superimposed modulations or consider the general case of superposition coding. In this work, we showed that the optimization of probabilities was often useful, but not always. However, superposition coding brings sometimes significant gains compared to superposition modulation, depending on the diameter of coverage area for the new layer of users.

This work can also be extended to two-dimensional constellations like M-QAM and other channel models. The maximization achievable rates using various transmission strategies can be performed also using the proposed algorithm based on alternative maximization with respect to symbol positions and the joint distribution of probability.

\section{Competing interests}

The authors declare that they have no competing interests.

\section{Author details}

${ }^{1}$ University Paris-Sud, UMR8506 Orsay, F-91405, France. ${ }^{2}$ CNRS, Gif-sur-Yvette, F-91192, France. ${ }^{3}$ Supelec, Gif-sur-Yvette, F-91192, 3 rue Joliot-Curie, 91192 Gif-sur-Yvette Cedex, France.

Received: 27 June 2013 Accepted: 20 October 2013 Published: 31 October 2013

\section{References}

1. TM Cover, Broadcast channels. IEEE Trans. Inform. Theory 18, 2-14 (1972)

2. PP Bergmans, Random coding theorem for broadcast channels with degraded components. IEEE Trans. Inform. Theory 19(2), 197-207 (1973)

3. PP Bergmans, A simple converse for broadcast channels with additive white Gaussian noise. IEEE Trans. Inform. Theory 20, 279-280 (1974)

4. RG Gallager, Capacity and coding for degraded broadcast channels. Probl. Infor. Transm. 10(3), 185-193 (1974)

5. G Imai, S Hirakawa, A new multilevel coding method using error correcting codes. IEEE Trans. Inform. Theory 23, 371-377 (1977)

6. G Ungerboeck, Channel coding with multilevel/phase signals. IEEE Trans. Inform. Theory 28, 55-67 (1982)

7. PP Bergmans, TM Cover, Cooperative broadcasting. IEEE Trans. Inform. Theory 20, 317-324 (1974)

8. European Telecommunications Standards Institute, EN 300 744: Digital Video Broadcasting (DVB) _ framing structure, channel coding and modulation for digital terrestrial television. (European Telecommunications Standards Institute, France, 2004-2006) 
9. European Telecommunications Standards Institute, ETSI TS 102: Digital Video Broadcasting (DVB) - system specifications for satellite services to handheld devices $(\mathrm{SH})$ below $3 \mathrm{GHz}$. (European Telecommunications Standards Institute, France, 2008), p. 585

10. H Meric, J Lacan, C Amiot-Bazile, F Arnal, ML Boucheret. Generic approach for hierarchical modulation performance analysis: application to DVB-SH, in Wireless Telecommunications Symposium (New York, 13-15 April 2011)

11. AR Calderbank, LH Ozarow, Nonequiprobable signaling on the Gaussian channel. IEEE Trans. Inform. Theory 36(4), 726-740 (1990)

12. D Sommer, G Fettweis. Shaping by non-uniform QAM for AWGN channels and applications using turbo coding, in ITG Conference Source and Channel Coding (Munich, Germany, 17-19 Jan 2000)

13. C Fragouli, RD Wesel, D Sommer, GP Fettweis, Turbo codes with non-uniform constellations. Proc. IEEE Int. Conf. Commun. 1, 70-73 (2001)

14. N Varnica, X Ma, A Kavcic, Capacity of power constrained memoryless AWGN channels with fixed input constellations. GLOBECOM 2, 1339-1343 (2002)

15. D Raphaeli, A Gurevitz, Constellation shaping for pragmatic turbo-coded modulation with high spectral efficiency. IEEE Trans. Commun. 52(3), 341-345 (2004)

16. SY LeGoff, BK Khoo, CC Tsimenidis, BS Sharif, Constellation shaping for bandwidth-efficient turbo-coded modulation with iterative receiver. IEEE Trans. Wireless Commun. 6(6), 2223-2233 (2007)

17. NH Ngo, SA Barbulescu, SS Pietrobon. Performance of nonuniform M-ary QAM constellation on nonlinear channels, in Australian Communications Theory Workshop (Australia, 2-4 Feb 2005)

18. J Zhang, D Chen, Y Wang. A new constellation shaping method and its performance evaluation in BICM-ID, in Vehicular Technology Conference Fall (VTC 2009-Fall) (Anchorage, AK, 20-23 Sept 2009)

19. M Valenti, X Xiang, Constellation shaping for bit-interleaved LDPC coded APSK. IEEE Trans. Commun. 60(10), 2960-2970 (2012)

20. C Huppert, M Bossert. On achievable rates in the two user AWGN broadcast channel with finite input alphabets, in ISIT (Nice, 24-29 June 2007)

21. TM Cover, JA Thomas, Elements of Information Theory, 2nd edn. (Wiley, Hoboken, 2006)

22. J Gledhill, P Macavock, R Miles, DVB-T: Hierarchical Modulation. (DVB, Geneva, 2000)

23. A Schertz, C Weck, Technical Review: Hierarchical Modulation-the Transmission of Two Independent DVB-T Multiplexes on a Single Frequency. (EBU, Switzerland, 2003)

24. V Singh. On superposition coding for wireless broadcast channels. Master's thesis, Royal Institute of Technology, Sweden (2005). www.ee. kth.se/php/modules/publications/reports/2005/R-SB-EX-0507.pdf

25. Z Mheich, P Duhamel, L Szczecinski, ML Alberi-Morel. Constellation shaping for broadcast channels in practical situations, in 19th European Signal Processing Conference (Barcelona, 29 Aug-2 Sept 2011)

26. Z Mheich, ML Alberi-Morel, P Duhamel, Optimization of unicast services transmission for broadcast channels in practical situations. Bell Labs Techn. J. 17(5-24) (2012)

27. Z Mheich, F Alberge, P Duhamel. On the efficiency of transmission strategies for broadcast channels using finite size constellations, in 21st European Signal Processing Conference (Marrakech, 9-13 Sept 2013)

28. TM Cover, Comments on broadcast channels. IEEE Trans. Inform. Theory 44(6), 2524-2530 (1998)

29. RE Blahut, Computation of channel capacity and rate-distortion functions IEEE Trans. Inform. Theory 18(4), 460-473 (1972)

30. K Yasui, T Matsushima. Toward computing the capacity region of degraded broadcast channel, in ISIT (Austin, TX, 13-18 June 2010)

31. DP Bertsekas, Nonlinear Programming, 2nd edn. (Athena Scientific, Nashua, 1999)

doi:10.1186/1687-1499-2013-254

Cite this article as: Mheich et al:: Achievable rates optimization for broadcast channels using finite size constellations under transmission constraints. EURASIP Journal on Wireless Communications and Networking 2013 2013:254.

\section{Submit your manuscript to a SpringerOpen ${ }^{\circ}$ journal and benefit from:}

- Convenient online submission

- Rigorous peer review

- Immediate publication on acceptance

- Open access: articles freely available online

- High visibility within the field

- Retaining the copyright to your article 